\title{
Vainshtein screening in bimetric cosmology
}

\author{
Marvin Lüben ${ }^{1,{ }^{*}}$ Angnis Schmidt-May, ${ }^{1, \dagger}$ and Juri Smirnov ${ }^{2,3, *}$ \\ ${ }^{1}$ Max-Planck-Institut für Physik (Werner-Heisenberg-Institut), Föhringer Ring 6, 80805 Munich, Germany \\ ${ }^{2}$ Center for Cosmology and AstroParticle Physics (CCAPP), The Ohio State University, \\ Columbus, Ohio 43210, USA \\ ${ }^{3}$ Department of Physics, The Ohio State University, Columbus, Ohio 43210, USA
}

(Received 11 March 2020; accepted 20 November 2020; published 16 December 2020)

\begin{abstract}
We demonstrate that the early Universe in bimetric theory is screened by the Vainshtein mechanism on a Friedmann-Lemaitre-Robertson-Walker background. The spin-2 mass serves as the cosmological Vainshtein scale in this case. This allows us to quantitatively address early Universe cosmology. In particular, in a global analysis, we study data from the cosmic microwave background radiation and local measurements of the Hubble flow. We show that bimetric cosmology resolves the discrepancy in the local and early-time measurements of the Hubble scale via an effective phantom dark energy component.
\end{abstract}

DOI: 10.1103/PhysRevD.102.123529

\section{INTRODUCTION}

Modifications and extensions to the theory of general relativity (GR) are highly restricted [1,2]. One possible direction is the addition of new degrees of freedom to the massless spin-2 field in a consistent manner. Ghost-free bimetric theory describes a massless and a massive spin-2 field with fully nonlinear (self-)interactions [3-18]. It contains both general relativity and massive gravity in certain parameter limits in which the massive or the massless field decouples, respectively. As such, bimetric theory fills a gap in the list of consistent field theories for massive and massless particles with spin up to 2 and represents an extended gravitational theory with a rich phenomenology.

Bimetric theory can address several open questions in modern cosmology. The theory gives rise to self-accelerating solutions where the interaction energy between the massive and massless spin-2 field acts as dynamical dark energy [19-26]. The modification of the gravitational potential (as compared to GR) affects the required dark matter abundance from galactic to galaxy cluster scales [27]. Also, the massive spin-2 field itself serves as a candidate for dark matter [28-30]. Despite these successes, the stability of perturbations around the cosmological background [25,26,31-35]

\footnotetext{
*mlueben@mpp.mpg.de

† angnissm@mpp.mpg.de

smirnov.9@osu.edu
}

Published by the American Physical Society under the terms of the Creative Commons Attribution 4.0 International license. Further distribution of this work must maintain attribution to the author(s) and the published article's title, journal citation, and DOI. Open access publication funded by the Max Planck Society. still poses an open problem. ${ }^{1}$ At early times, certain perturbations become large, rendering linear perturbation theory invalid.

In static systems with spherical symmetry an analogous behavior occurs [7,36-39]: Nonlinear effects in the perturbations are relevant and render the linear approximation invalid. This is the well-known Vainshtein screening mechanism [7] that restores GR in spacetime regions where the energy density is large (compared to the spin-2 mass). In massive gravity, this behavior is crucial because it cures the so-called van Dam-Veltman-Zakharov (vDVZ) discontinuity [5,6].

In this paper, we give a physical argument for the cosmological version of the Vainshtein mechanism. We find that the spin-2 mass sets the energy scale at which Vainshtein screening sets in. Above this energy scale, the energy density coming from the interaction of the massive and massless mode is suppressed. We show that the nonlinear massless and massive modes indeed decouple in this regime. It then becomes clear that the Universe transitions from a Vainshtein screened period at early times into a late-time de Sitter phase. We use this insight to discuss the instability of linear perturbations around the cosmological background in light of existing results.

The Vainshtein mechanism is also known to play a crucial role in scalar-tensor Galileon cosmologies [40]. It has been pointed out, that at early times the Galileon dynamics is governed by self-interactions, which results in a suppressed energy density relative to matter and radiation [41]. In this work we focus on an analogous effect in the framework of bimetric theory.

\footnotetext{
${ }^{1}$ Linear perturbation theory breaks down at a scale that can be pushed to arbitrary early times [35]. This is precisely the GR limit of the theory.
} 
We use our result to address a highly debated problem of the Lambda cold dark matter $(\Lambda \mathrm{CDM})$ model, the $H_{0}$-tension. As discussed in the literature [42-55], cosmic microwave background (CMB) observations tend to predict a lower Hubble scale than local measurements. We demonstrate that this tension can be resolved in a minimal realization of bimetric theory with only two more parameters than the $\Lambda \mathrm{CDM}$ model. These are the mass of the spin-2 field and its coupling constant to ordinary matter. We perform a fit to the CMB, Cepheid, supernova type Ia (SN Ia) and baryon-acoustic-oscillations (BAOs) observables and demonstrate that the tension with the current data are alleviated. Instead, all observables are within $1 \sigma$ error intervals. This is achieved via an effective phantom phase of the dark energy component in the redshift interval $1 \lesssim z \lesssim 10$. Finally, we comment on the compatibility between the different observables and argue that the $\Lambda \mathrm{CDM}$ and the $\beta_{0} \beta_{1} \beta_{4}$ models are distinguishable with future measurements.

\section{REVIEW OF BIMETRIC THEORY}

In this section, we review some technical aspects of the bimetric theory. Readers familiar with bimetric theory might want to jump directly to the next section. For a review on bimetric theory we refer to Ref. [56]. For an introduction to the broad field of massive gravity we refer to Refs. [16,57].

\section{A. Action and equations of motion}

The action of bimetric theory with standard model matter minimally coupled to one metric is $[9,10,15]$

$$
\begin{aligned}
S= & \frac{m_{g}^{2}}{2} \int \mathrm{d}^{4} x\left(\sqrt{-g} R(g)+\alpha^{2} \sqrt{-f} R(f)\right) \\
& -m_{g}^{2} \int \mathrm{d}^{4} x \sqrt{-g} V(g, f)+\int \mathrm{d}^{4} x \sqrt{-g} \mathcal{L}_{m}(g, \Phi),
\end{aligned}
$$

where $R(g)$ and $R(f)$ are the Ricci scalars of the metric tensors $g_{\mu \nu}$ and $f_{\mu \nu}$, respectively. The parameter $m_{g}$ is the Planck mass of $g_{\mu \nu}$ and $\alpha$ parametrizes the ratio to the Planck mass of $f_{\mu \nu}$. The interaction part of the Lagrangian has been derived prior to the bimetric formulation in $[9,10]$. The potential, whose structure is entirely fixed by the absence of ghost instabilities, reads

$$
V(g, f)=\sum_{n=0}^{4} \beta_{n} e_{n}\left(\sqrt{g^{-1} f}\right)
$$

in terms of the elementary symmetric polynomials $e_{n}$. The interaction parameters $\beta_{n}$ have mass-dimension two in our parametrization. Matter fields, collectively denoted by $\Phi$, minimally couple to the metric $g_{\mu \nu}$ via the generic matter Lagrangian $\mathcal{L}_{\mathrm{m}}$.
Varying the action (1) with respect to the metric tensors yields two sets of modified Einstein equations,

$$
G_{\mu \nu}^{g}+V_{\mu \nu}^{g}=\frac{1}{m_{g}^{2}} T_{\mu \nu}, \quad G_{\mu \nu}^{f}+V_{\mu \nu}^{f}=0,
$$

where $G_{\mu \nu}^{g}$ and $G_{\mu \nu}^{f}$ are the Einstein tensors of $g_{\mu \nu}$ and $f_{\mu \nu}$, respectively. The variations of the potential yield

$$
\begin{gathered}
V_{\mu \nu}^{g}=g_{\mu \lambda} \sum_{n=0}^{3}(-1)^{n} \beta_{n} Y_{(n) \nu}^{\lambda}\left(\sqrt{g^{-1} f}\right), \\
V_{\mu \nu}^{f}=f_{\mu \lambda} \sum_{n=0}^{3}(-1)^{n} \beta_{4-n} Y_{(n) \nu}^{\lambda}\left(\sqrt{f^{-1} g}\right),
\end{gathered}
$$

where the matrices $Y_{(n) \nu}^{\lambda}(S)$ are sums of different powers and contractions of the matrix $\sqrt{g^{-1} f}$ [11]. Since matter minimally couples to $g_{\mu \nu}$, the stress-energy tensor for $g_{\mu \nu}$ is given by

$$
T_{\mu \nu}=\frac{-2}{\sqrt{-g}} \frac{\delta \sqrt{-g} \mathcal{L}_{m}}{\delta g^{\mu \nu}}
$$

For usual matter (invariant under diffeomorphisms), stress energy is conserved

$$
\nabla_{\mu} T^{\mu \nu}=0
$$

where $\nabla_{\mu}$ is the covariant derivative compatible with $g_{\mu \nu}$. Due to the Bianchi identity $\nabla^{\mu} G_{\mu \nu}^{g}=0$, this implies an onshell constraint on the potential,

$$
\nabla^{\mu} V_{\mu \nu}^{g}=0,
$$

referred to as the Bianchi constraint.

\section{B. Proportional background}

The theory has a well-defined mass spectrum only around proportional backgrounds where the two metrics are related as $f_{\mu \nu}=c^{2} g_{\mu \nu}$ for some nonvanishing constant $c$ $[15,58]$. The solution is an Einstein background, $R_{\mu \nu}(f)=$ $R_{\mu \nu}(g)=\Lambda g_{\mu \nu}$, with a cosmological constant given by

$$
\begin{aligned}
\Lambda & =\beta_{0}+3 \beta_{1} c+3 \beta_{2} c^{2}+\beta_{3} c^{3} \\
& =\frac{1}{\alpha^{2}}\left(\frac{\beta_{1}}{c}+3 \beta_{2}+3 \beta_{3} c+\beta_{4} c^{2}\right) .
\end{aligned}
$$

The equality of the first and second line is required for the consistency of the vacuum solution. It is a quartic polynomial in $c$ and each root corresponds to a different Einstein solution to the bimetric field equations. 
The Fierz-Pauli mass of the massive spin-2 mode that propagates on the proportional background is given by

$$
m_{\mathrm{FP}}^{2}=\left(1+\frac{1}{\alpha^{2} c^{2}}\right) c\left(\beta_{1}+2 \beta_{2} c+\beta_{3} c^{2}\right),
$$

in terms of the bimetric parameters. For later use, let us introduce the shorthand notation $\bar{\alpha}=\alpha c$. In the rest of the paper, we will refer to $\bar{\alpha}, m_{\mathrm{FP}}$, and $\Lambda$ as the physical parameters of a solution [59].

\section{Friedmann-Lemaître-Robertson-Walker solutions}

To describe the Universe on large scales, we assume spacetime to be homogeneous and isotropic according to the cosmological principle. Both metrics take on Friedmann-Lemaître-Robertson-Walker (FLRW) form $[20,21,23]$,

$$
\begin{aligned}
& \mathrm{d} s_{g}^{2}=a(\eta)^{2}\left(-\mathrm{d} \eta^{2}+\mathrm{d} \vec{x}^{2}\right), \\
& \mathrm{d} s_{f}^{2}=b(\eta)^{2}\left(-(1+\mu(\eta))^{2} \mathrm{~d} \eta^{2}+\mathrm{d} \vec{x}^{2}\right),
\end{aligned}
$$

where we fixed the time-reparametrization invariance such that we work in conformal time $\eta$ of the $g$-metric. The scale factors $a(\eta)$ and $b(\eta)$ of the metrics $g_{\mu \nu}$ and $f_{\mu \nu}$ are functions of time only. The $f$-metric lapse $(1+\mu)$ parametrizes the relative twist between the coordinate systems of the two metrics. In this sense, it is similar to a Stückelberg field since it would be shifted by time reparametrizations of the metric $f_{\mu \nu}$. From now on, we suppress the $\eta$ dependence. For convenience, let us introduce the Hubble rate and the ratio of the scale factors as

$$
\mathcal{H}=\frac{\dot{a}}{a}, \quad y=\frac{b}{a},
$$

where the dot represents the derivative with respect to conformal time. The conformal and physical Hubble rates are related via $\mathcal{H}=a H$.

We assume the Universe to be filled with a perfect fluid with stress-energy tensor,

$$
T^{\mu \nu}=\left(\rho_{\mathrm{m}}+p_{\mathrm{m}}\right) u^{\mu} u^{\nu}+p_{\mathrm{m}} g^{\mu \nu},
$$

where $\rho_{\mathrm{m}}$ is the energy density and $p_{\mathrm{m}}$ the pressure of the matter fluid. They are related via the linear equation of state $w_{\mathrm{m}}=p_{\mathrm{m}} / \rho_{\mathrm{m}} \cdot u^{\mu}$ is the 4-velocity of the fluid. The conservation Eq. (7) leads to the continuity equation,

$$
\dot{\rho}_{\mathrm{m}}+3 \mathcal{H}\left(1+w_{m}\right) \rho_{\mathrm{m}}=0,
$$

which is solved by $\rho_{\mathrm{m}}=\rho_{\mathrm{m}, 0} a^{-3\left(1+w_{m}\right)}$, where $\rho_{\mathrm{m}, 0}$ is an integration constant. The physical scale factor is related to the redshift as $a=(1+z)^{-1}$.
The Bianchi constraint on the dynamical branch ${ }^{2}$ is solved by

$$
\mu=\frac{\dot{y}}{\mathcal{H} y}=\frac{y^{\prime}}{y}
$$

where we have introduced the derivative with respect to $e$-folds, ${ }^{\prime}=\mathrm{d} / \mathrm{d} \ln a$. On the dynamical branch, the modified Friedmann equations for $g_{\mu \nu}$ and $f_{\mu \nu}$ read

$$
\begin{gathered}
3 \mathcal{H}^{2}=a^{2}\left(\frac{\rho_{\mathrm{DE}}}{m_{g}^{2}}+\frac{\rho_{\mathrm{m}}}{m_{g}^{2}}\right), \\
3 \alpha^{2} \mathcal{H}^{2}=a^{2}\left(\frac{\beta_{1}}{y}+3 \beta_{2}+3 \beta_{3} y+\beta_{4} y^{2}\right) .
\end{gathered}
$$

Here, we defined the energy density coming from the potential as

$$
\frac{\rho_{\mathrm{DE}}}{m_{g}^{2}}=\beta_{0}+3 \beta_{1} y+3 \beta_{2} y^{2}+\beta_{3} y^{3}
$$

which can be interpreted as dynamical dark energy. Combining both Friedmann equations yields a quartic polynomial for $y$,

$$
\begin{gathered}
\alpha^{2} \beta_{3} y^{4}+\left(3 \alpha^{2} \beta_{2}-\beta_{4}\right) y^{3}+3\left(\alpha^{2} \beta_{1}-\beta_{3}\right) y^{2} \\
+\left(\alpha^{2} \beta_{0}-3 \beta_{2}+\frac{\alpha^{2} \rho_{\mathrm{m}}}{m_{g}^{2}}\right) y-\beta_{1}=0,
\end{gathered}
$$

that can be thought of determining $y$ as a function of $\rho_{\mathrm{m}}$. The polynomial has in general up to four real-valued roots, of which only one (the so-called finite branch) is physical $[24,26]$. On this branch, the ratio of scale factors evolves from $y=0$ in the early Universe (where $\rho_{\mathrm{m}}=\infty$ ), to a finite constant value $y=c$ in the asymptotic future (where $\rho_{\mathrm{m}}=0$, i.e., de Sitter space).

Taking the derivative with respect to $e$-folds and using the conservation Eq. (14), we can express $y^{\prime}$ as a function of $y$ as [22]

$$
y^{\prime}=\frac{3\left(1+\omega_{\mathrm{m}}\right) \alpha^{2} y^{2} \rho_{\mathrm{m}} / m_{g}^{2}}{\beta_{1}-3 \beta_{3} y^{2}-2 \beta_{4} y^{3}+3 \alpha^{2} y^{2}\left(\beta_{1}+2 \beta_{2} y+\beta_{3} y^{2}\right)},
$$

where $\rho_{\mathrm{m}}$ is a function of $y$ via Eq. (19). In terms of the dynamical mass parameter

\footnotetext{
${ }^{2}$ Another solution to the Bianchi constraint is the algebraic branch, where the ratio of the scale factors is fixed, $y=$ const. This branch was studied in the literature and found to be pathological; see, e.g., Refs. [26,31,60].
} 


$$
m_{\mathrm{eff}}^{2}=\frac{1+\alpha^{2} y^{2}}{\alpha^{2} y^{2}} y\left(\beta_{1}+2 \beta_{2} y+\beta_{3} y^{2}\right)
$$

we can rewrite Eq. (20) as

$$
\frac{y^{\prime}}{y}=\frac{\left(1+w_{\mathrm{m}}\right) \rho_{\mathrm{m}} / m_{g}^{2}}{m_{\mathrm{eff}}^{2}-2 H^{2}}
$$

In the following discussion we always assume an expansion history on the finite branch ${ }^{3}$ implying $m_{\mathrm{eff}}^{2}>2 H^{2}$ [61].

\section{VAINSHTEIN SCREENING}

The Vainshtein mechanism was discovered in systems, where due to a locally increasing gravitational field, GR is restored by nonlinear interactions $[7,37]$. We translate the analysis to the case where the gravitational field varies in time. By studying the cosmological background and linear perturbations we identify the energy scale at which nonlinearities become important. Due to our analogy this can be interpreted as a cosmological Vainshtein mechanism.

\section{A. The standard Vainshtein mechanism}

On the technical level, the Vainshtein screening effect is caused by the strong coupling of the longitudinal helicity- 0 mode of the massive spin-2 field.

In the case of a point source in bimetric theory, the critical radius below which the nonlinearities of the fields become strong enough is given by [38]

$$
r_{\mathrm{V}}=\left(\frac{r_{\mathrm{S}}}{m_{\mathrm{FP}}^{2}}\right)^{1 / 3}
$$

Here, the Schwarzschild radius for a source of mass $M$ is given by

$$
r_{\mathrm{S}}=\frac{M}{4 \pi m_{g}^{2}} .
$$

For a central, pointlike source (or on scales much larger than the massive object itself), the induced gravitational potential is then given by $[29,38,62,63]$

$$
\phi(r)= \begin{cases}-\frac{1}{m_{g}^{2}} \frac{1}{r} & r \ll r_{\mathrm{V}}, \\ -\frac{1}{m_{\mathrm{Pl}}^{2}}\left(\frac{1}{r}+\frac{4 \bar{\alpha}^{2}}{3} \frac{e^{-m_{\mathrm{FP}} r}}{r}\right) & r \gg r_{\mathrm{V}} .\end{cases}
$$

Inside the Vainshtein sphere, i.e., when $r \ll r_{\mathrm{V}}$, the gravitational potential is the same as in GR with Planck mass $m_{g}$. Outside the Vainshtein sphere, the massive spin-2 field propagates, contributing an attractive Yukawa term to the potential. On scales much larger than the Compton

\footnotetext{
${ }^{3}$ The finite branch is only well defined for $\beta_{1}>0$ [24]. Hence, in this paper we exclude models with $\beta_{1}=0$.
}

wavelength, $r \gg m_{\mathrm{FP}}^{-1}$, the Yukawa term is suppressed and the potential coincides with the one in GR, however with a different Planck mass given by $m_{\mathrm{Pl}}=\sqrt{1+\bar{\alpha}^{2}} m_{g}$, compared to the one inside the Vainshtein sphere. In this sense, there are two different scales on which GR is recovered, but with different Planck masses. Note that we neglect an asymptotic cosmological constant in Eq. (25).

Given a Schwarzschild geometry, on a technical level, the crucial indicator for the transition from the Vainshtein screened regime to the massive gravity regime is the radial, relative metric twist $\mu(r)$ [37,63]. This function drops off quickly outside the Vainshtein regime and can be used as a small parameter in perturbation theory. Inside the Vainshtein regime $\mu(r)$ becomes large and the perturbative expansion breaks down. On the nonlinear level, however, in this regime, the GR solution is recovered. We will show in the following, that the same behavior is present on the FLRW background and use the temporal metric twist function $\mu(\eta)$ introduced above to study when it becomes nonlinear.

For a source of finite size, the Vainshtein mechanism can be at work, once the radius within which the matter is concentrated is smaller than the Vainshtein radius itself. This indicates that there exists a minimal density for the Vainshtein mechanism. In the next section, we will elaborate on this observation and apply its logic to cosmology.

\section{B. The Vainshtein mechanism in cosmology}

In this section, we will explore possible implications of the standard Vainshtein mechanism for the Universe filled with a homogeneous energy density. From analogy with the Galileon cosmology [40,41], we expect that a cosmological Vainshtein regime would be present in bimetric theory, and restore a GR-like solution at early times and large densities in the Universe.

In particular, using properties of the solution for a static, spherically symmetric system around a compact source, we would like to answer the following question: What is the critical density $\rho_{\mathrm{c}}$ of a homogeneous mass distribution, for which the entire mass lies inside its own Vainshtein radius? We can only give a very rough estimate for this value since the standard expression for the Vainshtein radius is derived assuming that its value is larger than the radius of the source. In the following, we simply extend its definition to all possible configurations.

Let us thus consider a spherical, constant mass or energy distribution $\rho(r)=$ const. This distribution can be infinitely extended with radius $R=\infty$ or have a large but finite radius $R$; for our argument below, this will make no difference. The mass $M(r)$ enclosed within a distance $r<R$ from the center of the mass distribution is

$$
M(r)=\int_{0}^{r} \mathrm{~d}^{3} r^{\prime} \rho\left(r^{\prime}\right)=\rho \int_{0}^{r} \mathrm{~d}^{3} r^{\prime}=\frac{4 \pi}{3} r^{3} \rho .
$$


The Schwarzschild radius corresponding to this enclosed mass is

$$
r_{\mathrm{S}}=\frac{M(r)}{4 \pi m_{g}^{2}}=\frac{r^{3} \rho}{3 m_{g}^{2}} .
$$

The Vainshtein radius corresponding to the mass $M(r)$ is

$$
r_{\mathrm{V}}=\left(\frac{r_{\mathrm{S}}}{m_{\mathrm{FP}}^{2}}\right)^{\frac{1}{3}}=\left(\frac{\rho}{3 m_{g}^{2} m_{\mathrm{FP}}^{2}}\right)^{\frac{1}{3}} r .
$$

The mass within the radius $r$ fits precisely inside its own Vainshtein radius, when $r=r \mathrm{~V}$. This gives the value for the critical density,

$$
\rho_{\mathrm{c}}=3 m_{g}^{2} m_{\mathrm{FP}}^{2}
$$

For less dense systems with $\rho<\rho_{\mathrm{c}}$, the Vainshtein radius is smaller than the radius that encloses its corresponding mass. For denser systems with $\rho>\rho_{\mathrm{c}}$, the mass enclosed by a radius $r$ lies entirely inside its own Vainshtein radius.

We can now apply this result to cosmology, assuming that time evolution will not invalidate the arguments. Our reasoning suggests that there exists a critical value $\rho_{\mathrm{c}}$ for the homogeneous energy density $\rho(t)$ in the Universe, given by (29). Since $\rho(t)$ is time dependent and increases with redshift, there will be a point in time when it passes the value $\rho_{\mathrm{c}}$. Using Friedmann's equation, $H^{2}=\rho /\left(3 m_{g}^{2}\right)$, with $\rho$ being the total energy density of the Universe, this translates into a critical value for the Hubble rate,

$$
H_{\mathrm{c}}=m_{\mathrm{FP}}
$$

Moving backward in time, we reach the point $t_{\mathrm{c}}$ at which $H(t)$ passes the value $H_{\mathrm{c}}$. At this time, the energy density takes the value $\rho_{\mathrm{c}}$, for which its corresponding mass lies entirely inside its own Vainshtein radius.

For a homogenous matter distribution like in cosmology, the Vainshtein radius scales linearly with radius, $r_{\mathrm{V}} \sim r$. Therefore, if the energy density of the Universe is below its critical value, $\rho<\rho_{c}$ (or equivalently $H<m_{\mathrm{FP}}$ ), each Hubble patch is outside the Vainshtein regime. Analogously, for larger energy densities, $\rho>\rho_{c}$, and hence at early times, each Hubble patch lies within its Vainshtein region. Even though we apply a concept derived in a spherically symmetric setup to a homogenous energy distribution, the equations do not single out a preferred point in space. This is consistent with translational invariance of FLRW; our analogy does not spoil the cosmological principle. Instead, this allows for another, rather curious interpretation. In the expanding Universe, let us treat the big-bang singularity as a central source and measure the distance to it by the inverse Hubble scale $H^{-1}$. From this perspective, the profile of the energy density distribution is $\rho \sim H^{2} \sim r^{-2}$ according to Friedmann's equation.
Vainshtein vs Hubble scale

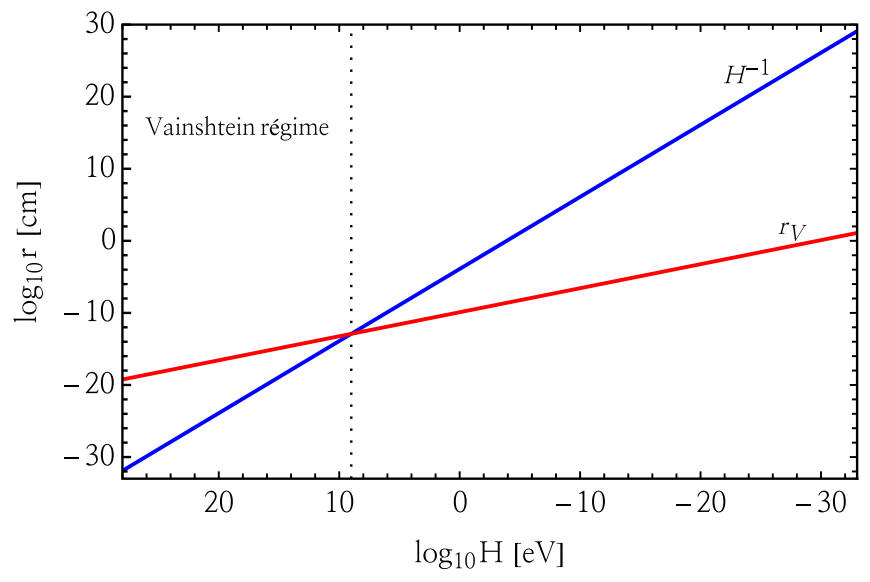

FIG. 1. For a spin-2 mass of $m_{\mathrm{FP}}=1 \mathrm{GeV}$, the size $H^{-1}$ of the Universe and its cosmological Vainshtein radius $r_{\mathrm{V}}$ are plotted as functions of the Hubble rate $H$. Since the Universe expands faster than its Vainshtein sphere, it unavoidably becomes larger than this sphere. That happens at the critical Hubble rate $H_{\mathrm{c}}=m_{\mathrm{FP}}$.

The corresponding Schwarzschild radius is $r_{\mathrm{S}}=H^{-1}$. Calculating the Vainshtein radius in this setup yields

$$
r_{\mathrm{V}}=\left(m_{\mathrm{FP}}^{2} H\right)^{-1 / 3},
$$

which of course leads to the same critical Hubble rate $H_{c}$ derived above as this is just the special case of the above analysis for $r=H^{-1}$.

In Fig. 1, we show the different scalings of the Vainshtein radius and of the size of the observable Universe as functions of the Hubble rate $H$. The cosmological Vainshtein radius scales as $r_{\mathrm{V}} \sim H^{-1 / 3}$ while the size of the observable Universe scales as $H^{-1}$. Therefore, at early times when the Hubble rate is large, the Vainshtein radius is larger than the size of a Hubble patch. At the critical value set by the spin- 2 mass, the former surpasses the latter, and at late times a Hubble patch is larger than its Vainshtein sphere.

In Fig. 2, the evolution of the Universe is schematically depicted, and can be interpreted as follows. The big-bang singularity serves as an analog of the central source in spherically symmetric systems. Moving away from the source, i.e., letting time pass, the evolution is expected to be governed by equations of motion equivalent to GR because we are inside the Vainshtein sphere. When the Hubble rate becomes comparable to the critical value, the Universe exits the Vainshtein regime and enters a transition phase where the evolution is governed by the bimetric field equations. Asymptotically in the future, the Universe approaches de Sitter space. In this sense, also at late times, GR is effectively recovered.

We emphasize again that we made simplifying assumptions about the validity of the expression for the 
Vainshtein (early times)
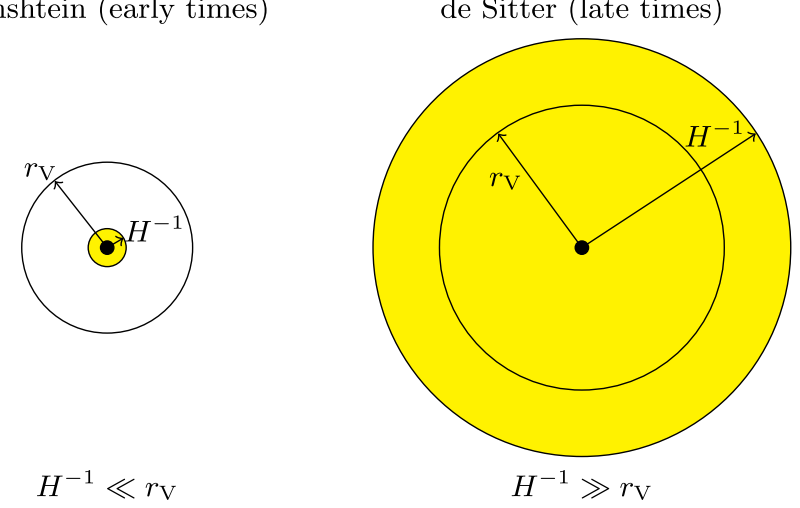

FIG. 2. Schematical depiction of the expansion of the Universe. Since the Universe expands faster than its Vainshtein radius, it leaves the Vainshtein sphere at the critical Hubble rate.

Vainshtein radius. ${ }^{4}$ Thus our arguments here can at most give very rough estimates for the critical values of cosmological quantities. Nevertheless, these approximate values are supported by results in cosmological perturbation theory; see Sec. IV B. Moreover, we observe interesting behavior of the solutions already at the background level, as we demonstrate in the next section.

\section{THE SPIN-2 MASS IN COSMOLOGY}

In the previous section we have identified $H_{\mathrm{c}}=m_{\mathrm{FP}}$ as the critical Hubble rate, at which the Universe transitions from a Vainshtein screened to an unscreened phase. In this section, we analyze how cosmological solutions to bimetric theory behave in the two regimes. In order to present explicit results, we use the $\beta_{0} \beta_{1} \beta_{4}$ model as a toy model for which we provide details in the Appendix.

\section{A. Vainshtein screening of background cosmology}

In this section, we demonstrate at the level of Friedmann's Eq. (16) that the background cosmology indeed transitions from the GR to the bimetric phase exactly at the energy scale $m_{\mathrm{FP}}$.

We start by discussing the scale factor ratio $y$. As discussed in Sec. II, on the finite branch the scale factor ratio evolves from zero at early times to a constant determined by Eq. (9) in the asymptotic future. More precisely, we can expand Eq. (19) for $\rho_{\mathrm{m}} / m_{g}^{2} \gg \beta_{n}$ to find

$$
\bar{y}=\bar{\beta}_{1}\left(\frac{\rho_{\mathrm{m}}}{m_{g}^{2}}\right)^{-1}+\mathcal{O}\left(\frac{\rho_{\mathrm{m}}}{m_{g}^{2}}\right)^{-2} .
$$

\footnotetext{
${ }^{4}$ Note that the standard derivation of the Vainshtein mechanism is restricted to scales $r$ which are much smaller than the Compton wavelength of the massive spin- 2 field, $r \ll m_{\mathrm{FP}}^{-1}$. When considering the entire Universe, the Vainshtein radius is $r_{V \text {,crit }}=m_{\mathrm{FP}}^{-1}$ and thus violates this condition.
}

Here we introduced the parameters $\bar{y}=\alpha y$ and $\bar{\beta}_{n}=\alpha^{-n} \beta_{n}$ for convenience [59]. Plugging this result into the expression for the dark energy density (18) yields

$$
\frac{\rho_{\mathrm{DE}}}{m_{g}^{2}}=\beta_{0}+\frac{3 \bar{\beta}_{1}^{2} m_{g}^{2}}{\rho_{\mathrm{m}}}+\mathcal{O}\left(\frac{\rho_{\mathrm{m}}}{m_{g}^{2}}\right)^{-2} .
$$

Since $\beta_{0}$ is a constant we thus find that $\rho_{\mathrm{m}} \gg \rho_{\mathrm{DE}}$ in the early Universe, as pointed out already in [20]. Hence, the energy density coming from the interaction between the spin-2 fields does not contribute to the Hubble rate at early times. This effect represents cosmological screening in close analogy to Galileon cosmology [41].

Next, we want to study when this screening sets in. As such, we determine the energy scale at which $\bar{y}^{\prime}$ is at its maximum. We therefore solve the equation $\bar{y}^{\prime \prime}=0$ and infer the corresponding Hubble rate. However, analytical solutions could not be found for this polynomial in the $\beta_{0} \beta_{1} \beta_{4}$ model due to its high degree. Instead, we solve $\bar{y}^{\prime \prime}=0$ for the two submodels with $\beta_{0}=0$ and $\beta_{4}=0$, respectively. The results in both cases are lengthy, but in the limit $\bar{\alpha} \ll 1$ we get the remarkably simple result

$$
H_{*} \simeq m_{\mathrm{FP}}
$$

as the critical Hubble rate at which $\bar{y}^{\prime \prime}=0$ for both submodels, up to an $\mathcal{O}(1)$ factor. $^{5}$

Let us provide a physical interpretation for the parameter $\bar{y}=\alpha y$. The nonlinear massless field $G_{\mu \nu}$ is given by [58]

$$
G_{\mu \nu}=g_{\mu \nu}+\alpha^{2} f_{\mu \nu}
$$

while the nonlinear massive field is not unique. On FLRW (11), the spatial components read $G_{i j}=a^{2}\left(1+\bar{y}^{2}\right) \delta_{i j}$. Hence, in the limit $\bar{y} \ll 1$ the nonlinear massless mode and the physical metric are aligned. This implies that the nonlinear massless and massive modes decouple. This observation is consistent with the decoupling of the linear massless and massive mode in the limit $\bar{\alpha} \ll 1[35,64]$. We conclude that $\bar{y}$ parametrizes the mixing of the massless and massive field on a FLRW background.

However, we also have to consider the temporal component of the nonlinear massless field that is given by $G_{00}=-a^{2}\left(1+\bar{y}^{2}(1+\mu)^{2}\right)$. The decoupling is not only

\footnotetext{
${ }^{5}$ Note that the limit $\bar{\alpha} \ll 1$ has very different meaning in both submodels [59]. Since we fix one of the interaction parameters, one of the physical parameters is not free, but depends on the other parameters. For the submodel where $\beta_{0}=0$ we get $\bar{\alpha}^{2}=$ $\Lambda /\left(3 m_{\mathrm{FP}}^{2}-\Lambda\right)$ while for the submodel with $\beta_{4}=0$ the relation is $\bar{\alpha}^{2}=m_{\mathrm{FP}}^{2} / \Lambda-1$; cf. Eq. (A1). Hence our limit in the former case implies $m_{\mathrm{FP}}^{2} \gg \Lambda$, while in the latter it implies $m_{\mathrm{FP}}^{2} \simeq \Lambda$. Consequently, for the $\beta_{0} \beta_{1}$ model there is only one energy scale involved. Expanding around $\bar{\alpha} \gg 1$ (i.e., $m_{\mathrm{FP}}^{2} \gg \Lambda$ ) instead results in $H_{*} \simeq\left(m_{\mathrm{FP}} \Lambda\right)^{1 / 3}$ as the critical Hubble rate at which $y^{\prime \prime}=0$. Only the $\beta_{0} \beta_{1}$ model gives rise to this behavior.
} 
controlled by $\bar{y}$, but also the Stückelberg field $\mu$. Hence, let us study the evolution of $\mu$. At late times, the matter-energy density $\rho_{\mathrm{m}}$ vanishes implying that $y^{\prime}$ vanishes and the Stückelberg field is small,

$$
\mu \rightarrow 0 \text { for } \eta \rightarrow+\infty
$$

cf. Eq. (15). At early times $\bar{y}$ approaches zero and the energy density diverges as $\rho_{\mathrm{m}} / m_{g}^{2} \sim \bar{\beta}_{1} / \bar{y}$. The Stückelberg field approaches the constant value,

$$
\mu \rightarrow 3\left(1+w_{\mathrm{m}}\right) \text { for } \eta \rightarrow-\infty,
$$

which is $\mathcal{O}(1)$. The Stückelberg field $\mu$ hence transitions from a large value at early times to a small value in the asymptotic future. Although this behavior does not spoil the decoupling, it is analogous to the effect in the Schwarzschild geometry. Here, $\mu$ asymptotes to a constant value when approaching the source [38].

In Fig. 3, we plot the Stückelberg field as a function of redshift $z$ in the $\beta_{0} \beta_{1} \beta_{4}$ model for three exemplary cases with different mixing angles $\bar{\alpha}$ and spin- 2 masses $m_{\mathrm{FP}}$ as displayed in the caption. The vertical lines represent the critical redshift at which $H=m_{\mathrm{FP}}$. Qualitatively we find that the Stückelberg field $\mu$ indeed starts to deviate from its asymptotic value as soon as the Hubble rate is of the order of $m_{\mathrm{FP}}$. We have explicitly checked this for various examples and find that the behavior is completely generic. ${ }^{6}$ Below the energy scale, $m_{\mathrm{FP}}$ the Stuickelberg field becomes small and enters its linear regime. We have already estimated the energy scale at which the Stückelberg field transitions from the linear to the nonlinear regime in Eq. (34).

In the limit of small $\bar{\alpha}$ we find the same expression for the critical Hubble rate as we found with the Vainshtein analogy. Away from the limit, the critical Hubble rate does not only depend on $m_{\mathrm{FP}}$ but also on $\bar{\alpha}$ and $\Lambda$. This can be seen in Fig. 3 because the inflection point $y^{\prime \prime}=0$ and the critical redshift are not exactly aligned. Our analogy with the local Vainshtein mechanism, hence, gives a rough estimate of the critical Hubble rate. The remarkable feature however is, that in the limit $\bar{\alpha} \ll 1$, the value of the critical Hubble rate at which the Stückelberg field becomes nonlinear and background cosmology is screened is set only by $m_{\mathrm{FP}}$ and becomes independent of $\bar{\alpha}$ and $\Lambda$.

\footnotetext{
${ }^{6}$ Note that for the case represented by the red line $\mu$ develops a peak. This happens only for models with $\beta_{0}<0$ as we checked explicitly. For submodels with $\beta_{0}=0, \mu$ does not develop a peak. Instead, the Stuickelberg field for these submodels always decreases monotonically in time. Since this behavior is already captured by the blue and green examples, we do not demonstrate that explicitly here. Let us only note that for all these submodels, $\mu$ starts to deviate from its asymptotic value $3\left(1+w_{\mathrm{m}}\right)$ as soon as the Hubble rate falls below the spin-2 mass.
}

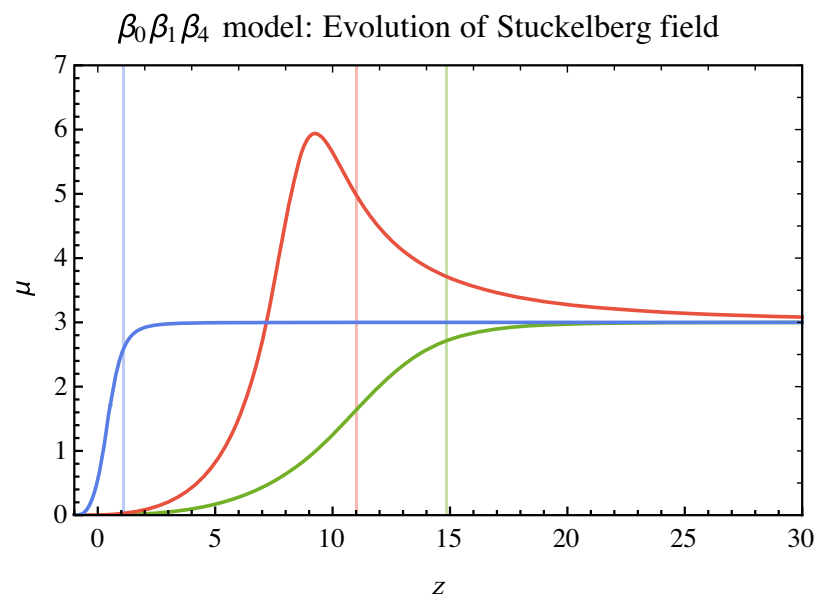

FIG. 3. The time evolution of the Stückelberg field $\mu$ as a function of redshift $z$. For all lines we set $\Lambda=0.7 H_{0}^{2}$ and $\rho_{\mathrm{m}, 0} / m_{g}^{2}=0.3 H_{0}^{2}$ as exemplary values. The bimetric parameters are $m_{\mathrm{FP}}=H_{0}$ and $\bar{\alpha}=0.5$ (blue line), $m_{\mathrm{FP}}^{2}=10 H_{0}^{2}$ and $\bar{\alpha}=2$ (red line), and $m_{\mathrm{FP}}^{2}=20 H_{0}^{2}$ and $\bar{\alpha}=0.01$ (green line). The vertical lines represent the redshift at which $H=m_{\mathrm{FP}}$, respectively. In each case, $\mu$ transitions from the early-time asymptotic value to 0 around the scale $m_{\mathrm{FP}}$.

Next, we study the Hubble rate. In Fig. 4 we show the Hubble rate as function of redshift, for several exemplary values. Again, the Hubble rate starts to deviate from the energy scale set by $\rho_{\mathrm{m}} / m_{g}^{2}$ when $H \sim m_{\mathrm{FP}}$, as indicated by the vertical lines. However, the deviations are suppressed by $\bar{\alpha}$. Therefore, the green $(\bar{\alpha}=0.01)$ and blue $(\bar{\alpha}=0.5)$ lines almost coincide. For the red line, $\bar{\alpha}=2$ is not small and the transition between the two phases can be seen

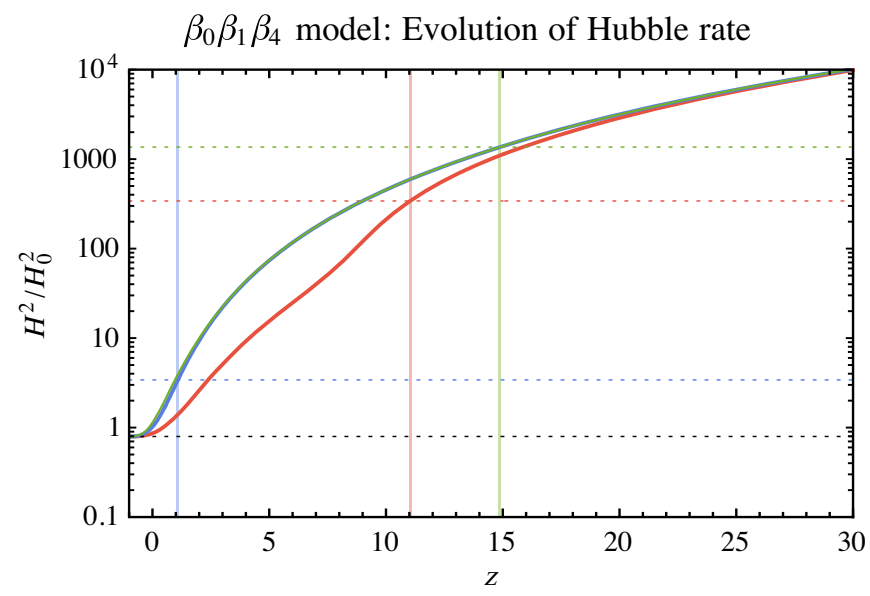

FIG. 4. The Hubble rate (normalized to $H_{0}$ ) as a function of redshift $z$ is shown. For all lines we set $\Lambda=0.7 H_{0}^{2}$ and $\rho_{\mathrm{m}, 0} / m_{g}^{2}=0.3 H_{0}^{2}$ as exemplary values. The bimetric parameters are $m_{\mathrm{FP}}=H_{0}$ and $\bar{\alpha}=0.5$ (blue line), $m_{\mathrm{FP}}^{2}=10 H_{0}^{2}$ and $\bar{\alpha}=2$ (red line), and $m_{\mathrm{FP}}^{2}=20 H_{0}^{2}$ and $\bar{\alpha}=0.01$ (green line). The vertical lines represent the redshift at which $H=m_{\mathrm{FP}}$, respectively. The horizontal dashed lines indicate $\left(m_{\mathrm{FP}} / H_{0}\right)^{2}$ (blue, red, green) and $\Lambda / 3 H_{0}^{2}$ (black). 
directly from the plot. Since the parameters that lead to the red line imply $\beta_{0}<0$, the energy contribution from the bimetric potential is negative for sufficiently large redshift. This implies that the Hubble rate is smaller than the energy scale set by $\rho_{\mathrm{m}} / m_{g}^{2}$.

To conclude, background cosmology of bimetric theory resembles GR when the massless and massive mode decouple. They decouple when $\bar{y} \ll 1$. This can be achieved by adjusting $\alpha \ll 1$, i.e., by going to the GR limit of the theory. Then, deviations from GR are suppressed at all redshifts. Alternatively, $\bar{y} \ll 1$ for $H \gg m_{\mathrm{FP}}$ as we have just shown. In this case, the energy density due to the nonlinear spin-2 interaction is screened and hence negligible above the same energy scale that we encountered in Sec. III B.

\section{B. Linear scalar perturbations and Vainshtein}

In this section, we connect our previous results to cosmological perturbations around the FLRW background in bimetric theory. Their analysis received a lot of attention in the literature $[25,26,32-35,65]$ and here we will summarize and rephrase the conclusions. It was found that the linear scalar perturbations in the WKB approximation are unstable on subhorizon scales during early times (for an expansion history on the finite branch). More precisely, they are stable as long as the dynamical bound [26]

$$
y^{\prime \prime}<\frac{y^{\prime}}{2 y} \frac{2 \mathcal{H} y^{\prime}\left(y^{\prime}-3 w_{m} y\right)-3 a^{2} \rho_{\mathrm{m}} y^{2}\left(w_{m}+1\right)\left(2 w_{m}+1\right)}{a^{2} \rho_{\mathrm{m}} y(w+1)+\mathcal{H}^{2} y^{\prime}}
$$

is satisfied. For models with $\beta_{2}=\beta_{3}=0$ this is equivalent to $y^{\prime \prime}<0$ [26].

Hence, the linear perturbations are unstable exactly when the background is screened. In other words, exactly when the Stückelberg field becomes nonlinear, linear perturbation theory breaks down. For the $\beta_{0} \beta_{1}$ and $\beta_{1} \beta_{4}$ model we already identified the energy scale at which $y^{\prime \prime}=0$. In Fig. 5, we show a schematic overview of the different regimes in bimetric cosmology. For completeness, we also compute at which energy scale the dynamical bound in Eq. (38) is violated for the remaining two-parameter models, $\beta_{1} \beta_{2}$ and $\beta_{1} \beta_{3}$. The expression for the critical Hubble rate is too long to display it here, but in the limit $\bar{\alpha} \ll 1$ we find $H_{*} \simeq m_{\mathrm{FP}}$ [up to an $\mathcal{O}(1)$ factor] ${ }^{7}$ as well.

The expansion history and the scalar perturbations are sensitive to the energy scale set by $m_{\mathrm{FP}}$ as we have explicitly demonstrated for all the two-parameter models. When the Hubble rate is of the order of the spin-2 mass, $H \sim m_{\mathrm{FP}}$, the Stückelberg field $\mu$ becomes nonlinear and the linear perturbations start to grow exponentially. At the

\footnotetext{
${ }^{7}$ For the $\beta_{1} \beta_{2}$ model, $H_{*}$ was already computed in Ref. [35] in the limit $\alpha \ll 1$, but not in terms of the physical parameters. They did not interpret their result as the spin-2 mass.
}

$$
\begin{array}{llc}
\text { Vainshtein/GR } & \text { Transition } & \text { de Sitter } \\
H^{2} \simeq \rho_{\mathrm{m}} / 3 m_{g}^{2} & H^{2} \simeq m_{\mathrm{FP}}^{2} & H^{2} \simeq \Lambda / 3 \\
\hline
\end{array}
$$

FIG. 5. Schematic summary of the cosmic evolution in bimetric theory.

same energy scale, the Universe becomes smaller than its own Vainshtein radius. Combining these results suggests that the Vainshtein mechanism is active also on a timedependent background like the FLRW and with spatially extended matter sources. Our result suggests that the scalar perturbations are cured by the Vainshtein mechanism.

Indeed, the stability of scalar perturbations around a FLRW background was studied in the literature. The authors of Ref. [65] solved the perturbation equations nonlinearly for early times. ${ }^{8}$ Their analysis identifies the spin-2 mass $m_{\mathrm{FP}}$ as the scale at which the Vainshtein mechanism kicks in and restores GR (for a generic model). In Ref. [66], on the other hand, the equations of motion were solved for an inhomogeneous mass distribution nonlinearly. Again, no instabilities were found. Both these results show that the instabilities are indeed an artifact of the linear approximation and that the Vainshtein mechanism is active also on a time-dependent background with a spatially extended source. In a different setting, the traditional Vainshtein mechanism was used to investigate the effect of early-time instabilities on structure formation; see Ref. [67].

\section{IMPACT ON THE $H_{0}$ TENSION}

Despite the huge success of general relativity describing gravitational systems on many different scales to enormous precision and in particular of the $\Lambda \mathrm{CDM}$ model, the latest data challenge the standard model of cosmology. Local observations of the Hubble flow are in good agreement with each other and constrain the value of the Hubble rate today to $h=0.7324 \pm 0.0174[42,52]$ where

$$
h=\frac{H_{0}}{100 \mathrm{~km} / \mathrm{s} / \mathrm{Mpc}}
$$

is the normalized Hubble rate today. Recently, a new measurement of the local Hubble rate was performed using megamasers [68]. This observation relies on independent distance determinations and thus has no overlap in systematic errors with previous studies. A Hubble rate value of

\footnotetext{
${ }^{8}$ In [65], spherically symmetric perturbations on subhorizon scales and on length scales smaller than the Compton wavelength $m_{\mathrm{FP}}^{-1}$ were studied. Furthermore, the background was assumed to be proportional, $\bar{g}_{\mu \nu}=y^{2} \bar{f}_{\mu \nu}$, with a time-dependent conformal factor $y$. This is only a solution to the equations of motion when both metrics couple to their own matter sector, which are proportional on shell.
} 
$h=0.739 \pm 0.030$ was found, in agreement with previous local measurements.

In the $\Lambda$ CDM model, $\mathrm{CMB}$ data from the Planck experiment, however, favor a value $h=0.6781 \pm 0.0092$ [46]. This causes an $\sim 3.4 \sigma$ tension with the Hubble rate value from local observations. While local measurements are quite sensitive to systematics $[54,55,69]$, the constraints from CMB measurements highly depend on the gravitational model [46]. Indeed, CMB data alone favor a dark energy component with a phantom equation of state, $w_{\mathrm{DE}}<-1[70]$.

\section{A. Phantom and negative dark energy}

Several mechanisms were proposed to alleviate the discrepancy in the value of $H_{0}$ inferred from late-time and CMB measurements. One possible direction is to lower the Hubble rate via late-time modifications of the $\Lambda \mathrm{CDM}$ model. This can be achieved by a dynamical dark energy component that grows as the Universe expands (phantom dark energy) or that changes sign at a certain redshift, i.e., with a negative energy density at sufficiently early times. Bimetric theory features both mechanisms depending on the parameters.

Models with a phantom equation of state should be treated with caution. Phantom energy violates the dominant energy condition [71] and causes a future spacetime singularity (big rip) [72-75]. For simple models that build on a single field (such as quintessence) a phantom equation of state implies the presence of a low-energy ghost [76]. There are ways to get around these issues. The big rip can be avoided if the equation of state varies in time and approaches -1 sufficiently fast [77-79]. Ghost condensation can stabilize the vacuum [80].

In contrast to these simple realizations, bimetric theory incorporates an effective phantom equation of state naturally. The dark energy density $\rho_{\mathrm{DE}}$ contained in Friedmann's Eq. (16) has the equation of state

$$
w_{\mathrm{DE}}=-1-\frac{\alpha^{2} y^{2}}{1+\alpha^{2} y^{2}} \frac{\left(1+w_{\mathrm{m}}\right) \rho_{\mathrm{m}}}{\rho_{\mathrm{DE}}} \frac{m_{\mathrm{eff}}^{2}}{m_{\mathrm{eff}}^{2}-2 H^{2}} .
$$

A cosmic expansion history on the finite branch implies $m_{\text {eff }}^{2}>2 H^{2}$ and hence for $\rho_{\mathrm{DE}}>0$ the equation of state is phantom, $w_{\mathrm{DE}}<-1$ [26]. At early times the asymptotic value of $w_{\mathrm{DE}}$ is either -1 (for models with $\beta_{0} \neq 0$ ) or $-2-w_{\mathrm{m}}$ (for models with $\beta_{0}=0$ ). In the asymptotic future (when $\rho_{\mathrm{m}} \rightarrow 0$ ) the equation of state approaches $w_{\mathrm{DE}} \rightarrow-1$ and the effect of the dynamical dark energy reduces to a cosmological constant. Hence, the big rip is avoided in bimetric theory.

Moreover, the effective dark energy component $\rho_{\mathrm{DE}}$ can change its sign. While in the infinite future the dynamical dark energy component approaches the value of the asymptotic cosmological constant, at early times $\rho_{\mathrm{DE}} / m_{g}^{2} \rightarrow \beta_{0}$ because $y \rightarrow 0$ on the finite branch. Hence, if

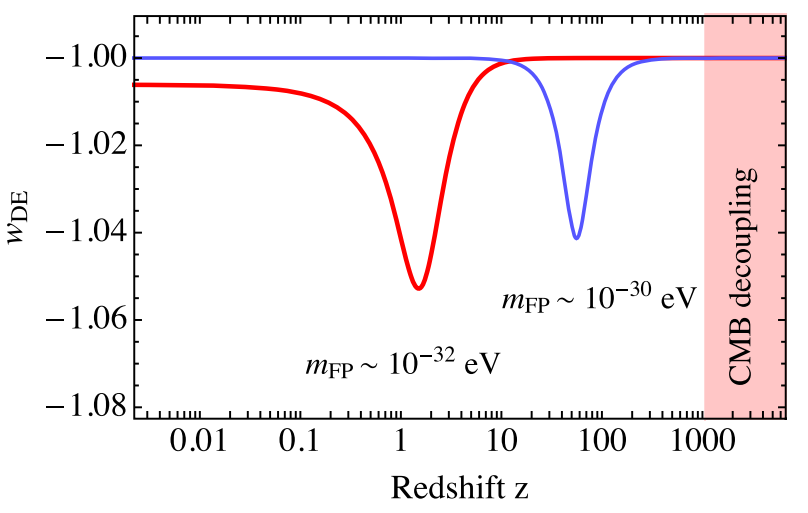

FIG. 6. The equation of state of the effective dark energy component. Two different scales for the spin-2 mass are chosen. The lighter corresponds to the Hubble scale today (red line) and the heavier to the Hubble scale at redshift $z \simeq 100$ (blue line).

$\beta_{0}<0$ the dark energy component is negative above a certain redshift. For the simple $\beta_{0} \beta_{1} \beta_{4}$ model this redshift is determined by $y=-\beta_{0} /\left(3 \beta_{1}\right)$ as follows from Eq. (18).

In Fig. 6, we show the time evolution of the dark energy equation of state for two different graviton masses. The phantom era of the cosmic evolution takes place when the size of the Universe is comparable to the wavelength of the graviton, i.e., $H(z) \simeq m_{\mathrm{FP}}$ and is related to the transition from the Vainshtein to the de Sitter regime.

The effective dark energy component violates the null energy condition (NEC) [81] allowing for a phantom equation of state while the sum of potential energy and matter stress energy satisfies the NEC. In bimetric theory, this does not imply the presence of a ghost mode due to the nontrivial interactions between the different modes that give rise to the effective phantom dark energy. On the other hand, the NEC violation manifests itself in linear perturbation theory as the gradient instability. However, as we argued earlier, it appears to be an artifact of the calculation and higher-order terms have to be taken into account due to the Vainshtein mechanism. It would be interesting to study the connection to ghost condensation.

In the following section we perform a fit to the data. We restrict ourselves to the study of the $\beta_{0} \beta_{1} \beta_{4}$ model. It is the simplest minimal bimetric model where all three physical parameters (mixing parameter $\bar{\alpha}$, spin-2 mass $m_{\mathrm{FP}}$, and cosmological constant $\Lambda$ ) are independent of each other. Already the authors of Ref. [52] studied phantom dark energy as a possible resolution to the $H_{0}$-tension. ${ }^{9}$ In particular, the bimetric $\beta_{0} \beta_{1}$ and $\beta_{1} \beta_{2}$ models were used as concrete models. They conclude that these models are driven into their GR limits and hence do not resolve the $H_{0}$-tension. However, for these two-parameter models of bimetric theory, not all the physical parameters are

\footnotetext{
${ }^{9}$ Additionally in Ref. [82], the inverse distance ladder method is discussed with the goal of testing the parameter region in bimetric theory, relevant for the $H_{0}$ tension.
} 
independent and the setup is too restricted. In particular, the fact that at small redshifts the equation of state is forced to be $w_{\mathrm{DE}} \simeq-1$ enforces a small mixing parameter $\bar{\alpha} \ll 1$. This is the GR limit of these models and implies an equation of state close to -1 at all redshifts. This is not the case for models where the three physical parameters are free as we will demonstrate in this section with the $\beta_{0} \beta_{1} \beta_{4}$ model. We find that solutions exist which feature $w_{\mathrm{DE}} \simeq-1$ at small redshifts but deviate from that value at intermediate redshifts. In the Appendix we collect the equations that we need for the analysis.

\section{B. Parametrization and scanning strategy}

To treat cosmological observables of late and early times on somewhat equal footings, we do the following. We approximate Friedmann's equation at late times by the Hubble law including the deceleration parameter $q=-\left(1+\dot{H} / H^{2}\right)$ as

$$
H(z)=H_{0}+(1+q) H_{0} z
$$

Observations of Cepheid variables constrain the Hubble rate parameter today to be $h=0.7324 \pm 0.0174$ [42]. The quoted value is derived from the combination of four independent Cepheid observables, which we will use to constrain the local Hubble rate.

Furthermore, we use observations of type Ia supernovae to constrain the deceleration parameter. We consider supernovae with redshifts $z<0.5$ such that the description in terms of the deceleration parameter is still valid. We find the best-fit value $q=-0.55 \pm 0.1$ which is consistent with the results of Refs. [83-85]. This approach projects a large number of SN Ia observables on one coarse-grained parameter. We choose this description to have a similar number of local and global observables. We restrict our analysis to the supernovae with moderate redshifts, as high-redshift supernova observations are subject to substantial luminosity uncertainties [86], and might be affected stronger by anisotropy effects [87].

One crucial point of the present analysis is the following. We assume that the physics that controls the inhomogeneities of the CMB in bimetric theory is identical to GR. This assumption is justified since the Vainshtein mechanism ensures that GR is restored at early times at the background level. For our analysis, we further assume, that small perturbations around this GR background are well described by the standard CMB perturbation theory.

The essence of the CMB physics can be well captured by the following coarse-graining method suggested in Ref. [52]. At the core of the analysis, there are only three physical observables, two of which are the shift parameters based on the comoving angular distance to the last scattering surface

$$
D_{A}\left(z^{*}\right)=\int_{0}^{z *} \frac{d z}{H(z)} \text { given that } \Omega_{K}=0
$$

where $z^{*}$ is the redshift at which decoupling happens, and the sound horizon

$r_{s}\left(z^{*}\right)=\int_{0}^{a *} \frac{c_{s} d a}{a^{2} H(a)}=\frac{1}{\sqrt{3}} \int_{0}^{a *} \frac{d a}{a^{2} H(a) \sqrt{1+\frac{3 \Omega_{\mathrm{b}}}{4 \Omega_{\gamma}} a}}$,

where $c_{s}$ is the speed of sound that depends on the energy density of baryons $\Omega_{\mathrm{b}}$ and of photons $\Omega_{\gamma}$. The physically constrained combinations are

(i) the angular distance normalized to the Hubble horizon at decoupling $\mathcal{R}=\sqrt{\Omega_{\mathrm{m}, 0}} H_{0} D_{A}\left(z^{*}\right)$,

(ii) and the principle multipole number $l_{A}=\pi \frac{D_{A}\left(z^{*}\right)}{r_{s}\left(z^{*}\right)}$. Here, $\Omega_{\mathrm{m}, 0}=\rho_{\mathrm{m}} /\left(3 m_{g}^{2} H_{0}^{2}\right)$ is the energy density today of nonrelativistic matter. The third parameter is the energy density of baryons at decoupling $\Omega_{\mathrm{b}} h^{2}$. We use the CMB compressed likelihood [46] with values $\left(\mathcal{R}, l_{A}, \Omega_{\mathrm{b}} h^{2}\right)=$ $(1.7382,301.63,0.02262)$, errors $(0.0088,0.15,0.00029)$, and the covariance matrix

$$
D_{\mathrm{CMB}}=\left(\begin{array}{ccc}
1.0 & 0.64 & -0.75 \\
0.64 & 1.0 & -0.55 \\
-0.75 & -0.55 & 1.0
\end{array}\right) .
$$

Note that in our analysis, we modify the analysis of Ref. [52] by adding a weight factor in the $\chi^{2}$ function. The weight factor $w_{p}$ multiplies the contribution of $l_{A}$ to the $\chi^{2}$ function, and thus takes into account the peak multiplicity in the CMB spectrum. We take $w_{p}=7$, as it corresponds to the number of peaks well resolved by the Planck experiment. This approach approximately models the statistical weight of the Planck data in comparison to the local observables.

The same physical scale of the CMB perturbations is imprinted in the matter power spectrum as the BAOs, and is accessible to us in the data of several surveys measuring galaxy distributions at different redshifts. The useful oblique parameter relevant for the computation of the matter distribution observable is the ratio of the sound horizon $r_{s}\left(z_{d}\right)$ and the spherical average of the angular scale and the redshift separation $d_{z}=r_{s}\left(z_{d}\right) / D_{V}(z)$, where

$$
D_{V}(z)=\left(D_{A}(z)^{2} \frac{z}{H(z)}\right)^{1 / 3},
$$

and $z_{d}$ is the drag epoch [88], the redshift at which the baryons are released from the Compton drag of the photons. We consider four experimental values of $d_{z}$ at different effective redshifts reported by the 6dF GS [89] 
TABLE I. The best-fit parameter values of the $\Lambda \mathrm{CDM}$ and the $\beta_{0} \beta_{1} \beta_{4}$ models. In both cases $\Omega_{\gamma}$ is irrelevant for late-time observations.

\begin{tabular}{lcc}
\hline \hline & $\Lambda \mathrm{CDM}$ & $\beta_{0} \beta_{1} \beta_{4}$ \\
\hline$h \times 100$ & $67.9_{-0.4}^{+0.4}$ & $72.3_{-0.4}^{+0.4}$ \\
$\bar{\alpha}$ & $\ldots$ & $0.13_{-0.03}^{+0.02}$ \\
$m_{\mathrm{FP}} / H_{0}$ & $\ldots$ & $0.59_{-0.13}^{+0.17}$ \\
$\Omega_{\Lambda}$ & $0.686_{-0.001}^{+0.001}$ & $0.707_{-0.002}^{+0.001}$ \\
$\Omega_{\mathrm{b}, 0}$ & $0.0488_{-0.0004}^{+0.0004}$ & $0.0430_{-0.0004}^{+0.0004}$ \\
\hline \hline
\end{tabular}

$d_{z}=0.34 \pm 0.02$ at $z_{\mathrm{eff}}=0.106 ;$ SDSS [90] $d_{z}=0.22 \pm$ 0.01 at $z_{\text {eff }}=0.15$; BOSS [91] $d_{z}=0.118 \pm 0.002$ at $z_{\text {eff }}=0.32$ and $d_{z}=0.072 \pm 0.001$ at $z_{\text {eff }}=0.57$.

Given this experimental input (i.e., from Cepheids, $\mathrm{SN}$ Ia, BAOs, and CMB), we perform a $\chi^{2}$ analysis. As a reference, we scan the $\Lambda \mathrm{CDM}$ model in the region $h=0.65-0.75, \Omega_{\Lambda}=0.6-0.8, \Omega_{\mathrm{b}, 0}=0-0.1$, and $\Omega_{\gamma, 0}=$ $0-10^{-2}$. The value of $\Omega_{\mathrm{m}, 0}$ is fixed by the flatness condition. For the $\beta_{0} \beta_{1} \beta_{4}$ model we scan over the same region and additionally have the free parameters $\bar{\alpha}=0-1$ and $\Omega_{m_{\mathrm{FP}}}=$ $m_{\mathrm{FP}}^{2} /\left(3 H_{0}^{2}\right)$ in the range $\Omega_{m_{\mathrm{FP}}}=0-50$. We perform at first a linear grid scan and refine the $\chi^{2}$ fit by a MetropolisHastings method.

\section{Numerical results}

In Table I, we show the best-fit values and one sigma intervals of the $\Lambda \mathrm{CDM}$ and the $\beta_{0} \beta_{1} \beta_{4}$ model. As expected, the $\Lambda \mathrm{CDM}$ model fit is poor. The best-fit value of $\chi^{2} \approx 14$ with 7 degrees of freedom suggests an $\sim 3 \sigma$ tension of the global fit. The error intervals are derived from projections on the one-dimensional subspaces of the likelihood function.

In contrast to this, when the fit is performed in the $\beta_{0} \beta_{1} \beta_{4}$ model, the fit is improved and $\chi^{2} \approx 3$. Given that we have only two additional fit parameters, resulting in 5 degrees of freedom, this corresponds to an excellent fit value, indicating that all observables are within the $1 \sigma$ error range. Another measure of the improvement is the $\Delta \chi^{2} /$ d.o.f., which in this case is $\sim 5$. We conclude that the given dataset favors the $\beta_{0} \beta_{1} \beta_{4}$ model.

In Fig. 7, we show the $\chi^{2}$ as a function of $H_{0}$ for the $\Lambda \mathrm{CDM}$ and the $\beta_{0} \beta_{1} \beta_{4}$ model. We find, that the alternative time evolution of the Hubble rate in bimetric theory can accommodate the CMB observables, and a larger $H_{0}$ value today, than the $\Lambda$ CDM scenario, while being consistent with the BAO observations. The favored Fierz-Pauli mass in the considered bestfit interval is $m_{\mathrm{FP}} \approx\left(4 \times 10^{-33}-7 \times 10^{-33}\right) \mathrm{eV}$, which is consistent with cluster lensing [27] and other constraints [64].

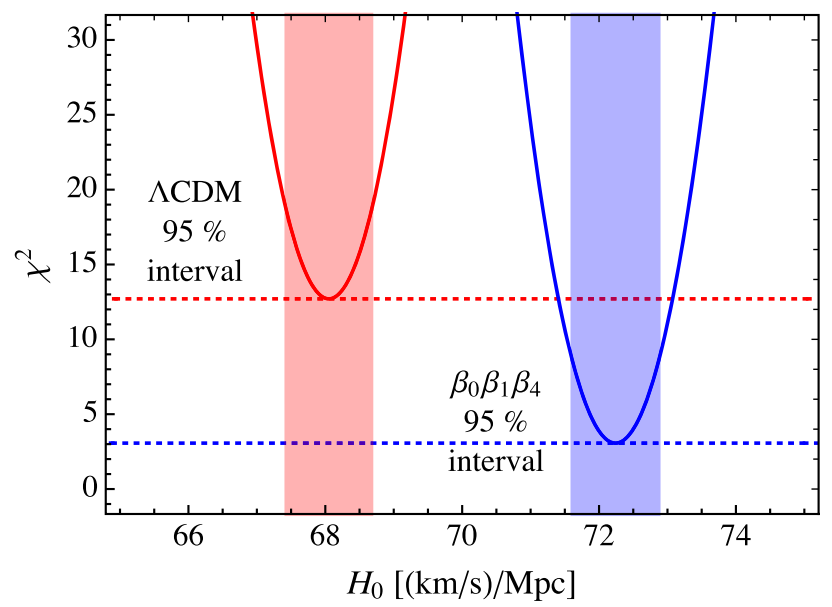

FIG. 7. The $\chi^{2}$ functions and $95 \%$ confidence intervals of $H_{0}$, around the best-fit points of the $\Lambda \mathrm{CDM}$ model and the $\beta_{0} \beta_{1} \beta_{4}$ realization of bimetric theory. The fit improvement is substantial, with $\Delta \chi^{2} /$ d.o.f. $\approx 5$.

\section{Discussion of results}

Now we discuss the results of the statistical analysis and underlying datasets. First note that the parameters at the best-fit point imply $\beta_{0}>0$ and hence $\rho_{\mathrm{DE}}>0$; cf. Eq. (A1a). Thus dark energy is always phantom and positive. In Fig. 8, we show the equation of state of the effective dark energy component in the $\beta_{0} \beta_{1} \beta_{4}$ model at the minimum of the $\chi^{2}$ function (solid line) and the $1 \sigma$ intervals (dashed lines). The values are in agreement with current experimental bounds [92].

The phantom behavior is most pronounced in the redshifts interval between $z \sim 1$ and $z \sim 10$. This is when the Hubble rate is of the order of the spin- 2 mass. The general behavior is the following. The spin-2 mass controls at which redshifts the equation of state significantly deviates from -1 , while the mixing parameter $\bar{\alpha}$ controls the magnitude of the deviation. To be precise, it is the value

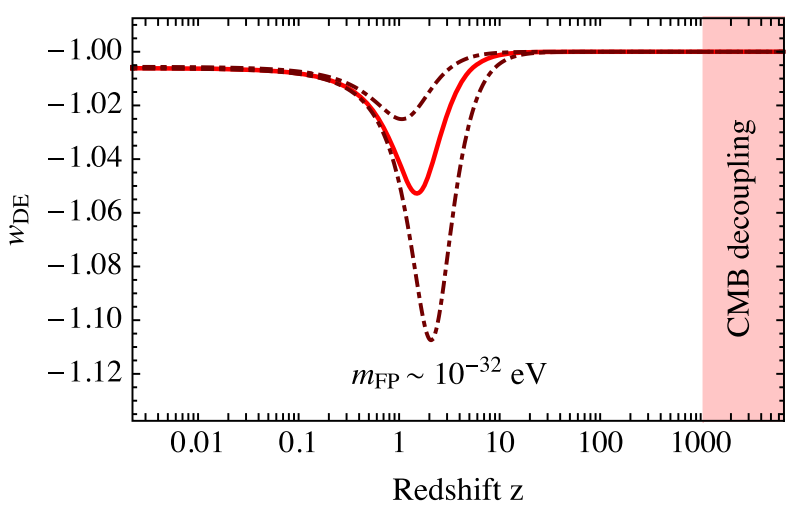

FIG. 8. The equation of state of the effective dark energy component. The minimum of the $\chi^{2}$ function in the bimetric model corresponds to the red line; see Table I. The dashed lines indicate the best-fit parameter intervals. 
of $\beta_{0}$ that controls the deviation. If its value is close to zero, the equation of state significantly deviates from -1 . On the other hand, if $\beta_{0}$ is positive and far away from zero, the equation of state is close to -1 at all times. Note that in order to achieve a value $\beta_{0}$ close to zero requires a nonzero mixing parameter $\bar{\alpha}$; cf. Eq. (A1a). In the GR limit $\bar{\alpha} \ll 1$ the phantom era is absent. The freedom to allow for large spin-2 masses and thus shifting the phantom behavior to larger $z$ while keeping $\bar{\alpha}$ finite to yield a significant phantom era is not possible in the more restricted twoparameter models [52].

The phantom behavior of the dynamical dark energy component lowers the Hubble rate for redshifts where it is still non-negligible, i.e., most importantly for $z \sim \mathcal{O}(1)$, as compared to a cosmological constant. The lowered Hubble rate increases the value of the integral in $D_{A}\left(z^{*}\right)$ which is compensated by a larger value of $H_{0}$ to arrive at the same value of $D_{A}\left(z^{*}\right)$ as desired. Since the value of $r_{s}\left(z^{*}\right)$ remains unchanged by late-time modifications, the value of $l_{A}$ remains unchanged. However, within $\mathcal{R}$ the larger value of $H_{0}$ must be compensated by a smaller value of $\Omega_{\mathrm{m}, 0}$ and consequently a larger value of $\Omega_{\Lambda}$. This is indeed the case (see Table I). ${ }^{10}$

For the $\Lambda$ CDM model a smaller value of $\Omega_{\mathrm{m}, 0}$ is disfavored by local measurements as well as the CMB because the matter-dark-energy equality is shifted to higher redshifts, also affecting the value of $l_{A}$. To see that bimetric cosmology with a smaller $\Omega_{\mathrm{m}, 0}$ is compatible with local experiments, we compare the prediction of the $\beta_{0} \beta_{1} \beta_{4}$ model (red solid line) to the four measurements of BAO observables in Fig. 9. For a better resolution, we normalized the plot to the $\Lambda \mathrm{CDM}$ prediction (dashed line). We can also see that, given the current experimental precision, BAOs cannot distinguish the predictions of the bimetric $\beta_{0} \beta_{1} \beta_{4}$ model from predictions of $\Lambda \mathrm{CDM}$ at their best-fit points. However, baryon acoustic oscillations might provide a test of bimetric cosmology at the best-fit point found in this section. In the near future the DESI instrument $[93,94]$ will provide a new dataset of BAO observations at multiple redshifts and even more advanced experiments will push to larger redshifts [95]. A big advantage of DESI will be that BAO data will be obtained at multiple redshifts with the same instrument, thus avoiding the problem of different systematic errors among the instruments. With the newly collected data, this question should be reexamined. In particular with an estimated accuracy improvement by a factor of 2 , as predicted in Ref. [96], the $\beta_{0} \beta_{1} \beta_{4}$ model at its best-fit point will be distinguishable from $\Lambda \mathrm{CDM}$.

Moreover, measurements of supernovae at high redshifts might allow us to distinguish the bimetric $\beta_{0} \beta_{1} \beta_{4}$ model from the $\Lambda \mathrm{CDM}$ model if the $H_{0}$-tension should be alleviated within bimetric theory solely due to the phantom phase. Although we only included supernovae with redshift

\footnotetext{
${ }^{10}$ We are thankful to E. Mörtsell for making this point clear.
}

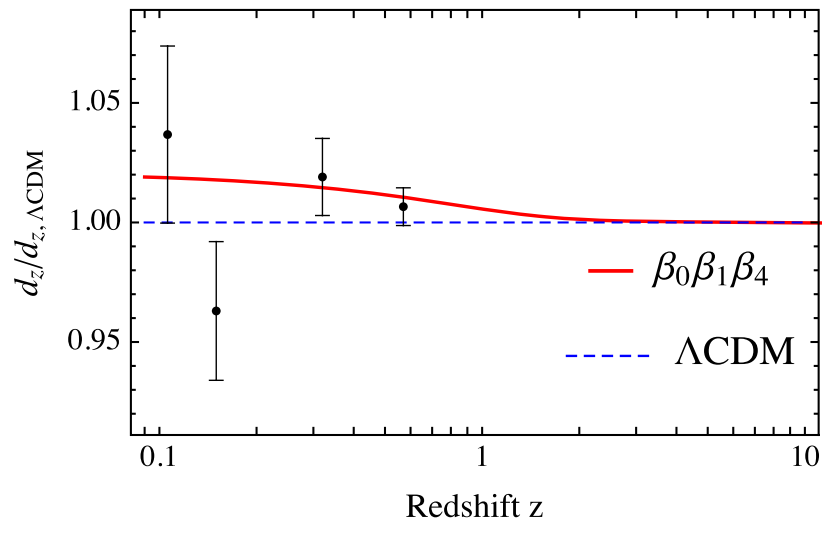

FIG. 9. The ratio of the baryon-acoustic-oscillation parameters normalized to the $\Lambda \mathrm{CDM}$ prediction $d_{z} / d_{z, \Lambda \mathrm{CDM}}$ in the $\beta_{0} \beta_{1} \beta_{4}$ model and the $\Lambda \mathrm{CDM}$ as a function of the cosmological redshift. Superposed are four measurement points at different redshifts.

$z<0.5$ in our analysis, our best-fit point is consistent also with supernovae measurements at higher redshifts [59] given the current experimental precision $[86,87]$.

When discussing the significance of the Hubble tension, a word of caution is in order. So far we have taken the local determinations of the Hubble rate at face value with the quoted uncertainties, which are reported to be at the 2\%-3\% level. However, the distance determination with the Cepheid observables is known to be subject to systematic errors, which are hard to control; see for example Ref. [97].

One important effect is the so-called blending effect and is based on the fact that the spatial resolution of our instruments gets worse with distance. Thus observations of Cepheids that are farther away are more likely to pick up light from unresolved background sources. This leads to systematically larger luminosities for more distant objects. This effect tends to increase the reconstructed local Hubble rate, which is consistent with the sign of the observed discrepancy. Taking the blending effect into account would increase the uncertainty to the $\sim 5 \%$ level $[98,99]$. Even though this would not resolve the Hubble tension, without proper control of the systematic error, we cannot make strong statements about the true statistical significance of this anomaly.

\section{SUMMARY AND DISCUSSION}

In the first part of this paper, we discussed the signatures of the Vainshtein mechanism in cosmology. We found that the Universe becomes larger than its own Vainshtein radius at the critical Hubble rate

$$
H_{\mathrm{c}}=m_{\mathrm{FP}}
$$

For earlier times, each Hubble patch lies within its own Vainshtein regime and hence is screened. We found the same scale appearing on the level of Friedmann's equation 
and linear perturbations. In particular, nonlinear massless and massive modes decouple at early times, i.e., for $H \gg m_{\mathrm{FP}}$. Further, the Stückelberg field becomes large and hence nonlinear above the same scale. Finally, linear perturbation around the FLRW background is unstable and grows exponentially when $H \gg m_{\mathrm{FP}}$. We concluded that the early Universe is screened by the cosmological analog of the Vainshtein mechanism. When studying perturbative phenomena in the early Universe, nonlinearities have to be taken into account.

We assumed that these nonlinearities are such that the massive mode becomes strongly coupled and can be integrated out to restore GR, as demonstrated in [65]. This allows us to study perturbative phenomena, such as the formation of the CMB.

Under this assumption (that CMB physics is the same as in GR due to the Vainshtein mechanism), we performed a global fit to the CMB observables and the local measurements on Cepheid variables, SN Ia observations of the cosmic acceleration, and baryon acoustic oscillations. We found that due to the phantom equation of state of the effective dark energy component in the redshift interval between $z \sim 1$ and $z \sim 10$ the reported tension between the local and CMB determination of the Hubble scale can be resolved.

We discussed some potential shortcomings of our statistical analysis. In particular the blending effect in Cepheid measurements and the high-redshift supernovae should be taken into account. However, we do not expect these to dramatically change our result as we discussed. Instead, if the $H_{0}$-tension is real and statistically significant, bimetric theory appears as an experimentally favored, consistent, and theoretically well-motivated alternative to GR. Whether data eventually favor bimetric theory also over other gravitational theories, remains an open question.

We showed that current data from BAOs cannot distinguish bimetric cosmology from $\Lambda \mathrm{CDM}$. However, in the near future, a strong improvement in sensitivity and systematic uncertainty will likely change this situation. Under the requirement that the $H_{0}$-tension is alleviated solely due to the bimetric phantom dark energy, the $\beta_{0} \beta_{1} \beta_{4}$ model will be distinguishable from the $\Lambda \mathrm{CDM}$ model in the near future.

A next step would be a global fit to all current data. In particular, instead of the coarse-grained methods used in our analysis the whole datasets should be used for studying the statistical significance of the tension and its alleviation within bimetric theory. Another direction is a nonlinear study of the cosmological perturbations and of the Vainshtein mechanism. Within an entirely bimetric framework, the constraints from the CMB should be derived to explicitly check our assumption a posteriori. Also, cosmic structure formation should be addressed entirely within bimetric theory as it probes redshifts where the phantom era occurs.

\section{ACKNOWLEDGMENTS}

We would like to thank Christopher Hirata, Florian Niedermann, Moritz Platscher, Anna Porredon, Fiorenzo Vincenzo, Bei Zhou, and especially Edvard Mörtsell for valuable discussions and helpful comments on the manuscript. This work is supported by a grant from the MaxPlanck-Society. J. S. is largely supported by a Feodor Lynen Fellowship from the Alexander von Humboldt Foundation. The work of M. L. is supported by a grant from the Max Planck Society.

\section{APPENDIX: EXPLICIT EXPRESSIONS FOR THE $\beta_{0} \beta_{1} \beta_{4}$ MODEL}

Throughout the paper we used the $\beta_{0} \beta_{1} \beta_{4}$ model that is defined by setting $\beta_{2}=\beta_{3}=0$ to provide an explicit example. In this Appendix, we report the exact expressions and discuss some features of the model. For details, we refer to [59].

First, let us find the relation between the interaction and physical parameters. The background Eq. (9) is a cubic polynomial in $c$ and gives rise to the three real-valued roots. Each root describes a vacuum of the $\beta_{0} \beta_{1} \beta_{4}$ model with different spin-2 mass, mixing angle, and cosmological constant. However as discussed in Ref. [59], only one of the vacua is physical. Therefore, the vacuum Eqs. (9) and (10) imply the following unique relation between the interaction parameters and the physical parameters,

$$
\begin{aligned}
\beta_{0} & =\frac{-3 \bar{\alpha}^{2} m_{\mathrm{FP}}^{2}+\left(1+\bar{\alpha}^{2}\right) \Lambda}{1+\bar{\alpha}^{2}}, \\
\alpha^{-1} \beta_{1} & =\frac{\bar{\alpha}}{1+\bar{\alpha}^{2}} m_{\mathrm{FP}}^{2}, \\
\alpha^{-4} \beta_{4} & =\frac{-m_{\mathrm{FP}}^{2}+\left(1+\bar{\alpha}^{2}\right) \Lambda}{\bar{\alpha}^{2}\left(1+\bar{\alpha}^{2}\right)} .
\end{aligned}
$$

The physical parameters are not completely free but have to satisfy the Higuchi bound $m_{\mathrm{FP}}^{2}>3 \Lambda / 2$ to ensure unitarity [100]. In the following, we still express all equations in terms of the interaction parameters $\beta_{n}$ for brevity but they should be understood as being functions of the physical parameters. Furthermore, we rescale $\bar{y}=\alpha y$ for brevity.

Setting $\beta_{2}=\beta_{3}=0$, Eq. (19) reduces to

$$
\bar{\beta}_{4} \bar{y}^{3}-3 \bar{\beta}_{1} \bar{y}^{2}-\left(\beta_{0}+\frac{\rho_{\mathrm{m}}}{m_{g}^{2}}\right) \bar{y}+\bar{\beta}_{1}=0,
$$

where $\bar{\beta}_{n}=\alpha^{-n} \beta_{n}$ for brevity. This polynomial has up to three real-valued roots that yield $y$ as a function of $\rho_{\mathrm{m}}$. For a given set of parameters, only one of these solutions corresponds to the finite branch. Since the expressions are quite lengthy and not enlightening, we do not show them 
explicitly here. The finite branch solution must satisfy $0 \leq \bar{y} \leq \bar{\alpha}$ which allows us to pick the finite branch solution numerically. Hence, we can express

$$
\mu=\frac{y^{\prime}}{y}
$$

either analytically (but lengthy) or numerically as a function of matter-energy density $\rho_{\mathrm{m}}$ and consequently as a function of redshift $z$ only. We used that for producing the exemplary plots in Fig. 3.

Next, we want to find the Hubble rate as a function of redshift $z$ only. For the $\beta_{0} \beta_{1} \beta_{4}$ model the Hubble rate reads

$$
3 H^{2}=\beta_{0}+3 \bar{\beta}_{1} \bar{y}+\frac{\rho_{\mathrm{m}}}{m_{g}^{2}}=\frac{\bar{\beta}_{1}}{\bar{y}}+\bar{\beta}_{4} \bar{y}^{2}
$$

where the interaction parameters $\bar{\beta}_{n}$ are understood as functions of the physical parameters and $\bar{y}$ as the finite branch solution to Eq. (A2) (either analytically or numerically). We used the result for drawing the plot in Fig. 4 and for the data analysis in Sec. V.

Let us collect some more details for the data analysis with the $\beta_{0} \beta_{1} \beta_{4}$ model. Evaluating Friedmann's equation today yields a relation among the parameters of the model,

$$
3 H_{0}^{2}=\beta_{0}+3 \bar{\beta}_{1} \bar{y}_{0}+\frac{\rho_{\mathrm{m}, 0}}{m_{g}^{2}},
$$

where the subscript 0 indicates the value of the quantity at present time. We used this relation to eliminate $\Omega_{m, 0}$ in terms of the other parameters.

In the data analysis, we constrained the deceleration parameter $q$ that is derived from Friedmann's equation. We can express the definition more explicit in terms of bimetric parameters as

$$
1+q=-\frac{\dot{H}}{H^{2}}=\frac{1}{2 H^{2}} \frac{\mathrm{d} H^{2}}{\mathrm{~d} \bar{y}} \bar{y}^{\prime}
$$

where $\bar{y}^{\prime}=\dot{\bar{y}} / H$ is given by Eq. (20) and

$$
\frac{\mathrm{d} H^{2}}{\mathrm{~d} \bar{y}}=-\frac{\bar{\beta}_{1}}{3 \bar{y}^{2}}+\frac{2}{3} \bar{\beta}_{4} \bar{y}
$$

Again, with $\bar{y}$ understood as the finite branch solution, $q$ is a function of redshift only. For the data analysis, we used the constraints on $q_{0}$ (i.e., at $z=0$ ) to find the favored values of the physical parameters. Note that Eq. (A6) holds for any bimetric (sub)model.
[1] D. Lovelock, The Einstein tensor and its generalizations, J. Math. Phys. (N.Y.) 12, 498 (1971).

[2] D. Lovelock, The four-dimensionality of space and the einstein tensor, J. Math. Phys. (N.Y.) 13, 874 (1972).

[3] W. Pauli and M. Fierz, On relativistic field equations of particles with arbitrary spin in an electromagnetic field, Helv. Phys. Acta 12, 297 (1939).

[4] M. Fierz and W. Pauli, On relativistic wave equations for particles of arbitrary spin in an electromagnetic field, Proc. R. Soc. A 173, 211 (1939).

[5] H. van Dam and M. J. G. Veltman, Massive and massless Yang-Mills and gravitational fields, Nucl. Phys. B22, 397 (1970).

[6] V. I. Zakharov, Linearized gravitation theory and the graviton mass, JETP Lett. 12, 312 (1970).

[7] A. I. Vainshtein, To the problem of nonvanishing gravitation mass, Phys. Lett. 39B, 393 (1972).

[8] D. G. Boulware and S. Deser, Can gravitation have a finite range?, Phys. Rev. D 6, 3368 (1973).

[9] C. de Rham and G. Gabadadze, Generalization of the Fierz-Pauli action, Phys. Rev. D 82, 044020 (2010).

[10] C. de Rham, G. Gabadadze, and A. J. Tolley, Resummation of Massive Gravity, Phys. Rev. Lett. 106, 231101 (2010).

[11] S. F. Hassan and R. A. Rosen, On non-linear actions for massive gravity, J. High Energy Phys. 07 (2011) 009.
[12] S. F. Hassan and R. A. Rosen, Resolving the Ghost Problem in Nonlinear Massive Gravity, Phys. Rev. Lett. 108, 041101 (2011).

[13] S. F. Hassan, R. A. Rosen, and A. Schmidt-May, Ghostfree massive gravity with a general reference metric, J. High Energy Phys. 02 (2012) 026.

[14] S. F. Hassan and R. A. Rosen, Confirmation of the secondary constraint and absence of ghost in massive gravity and bimetric gravity, J. High Energy Phys. 04 (2012) 123.

[15] S. F. Hassan and R. A. Rosen, Bimetric gravity from ghostfree massive gravity, J. High Energy Phys. 02 (2012) 126.

[16] C. de Rham, Massive gravity, Living Rev. Relativity 17, 7 (2014).

[17] C. de Rham, L. Heisenberg, and R.H. Ribeiro, On couplings to matter in massive (bi-)gravity, Classical Quantum Gravity 32, 035022 (2015).

[18] C. de Rham, L. Heisenberg, and R. H. Ribeiro, Ghosts and matter couplings in massive gravity, bigravity and multigravity, Phys. Rev. D 90, 124042 (2014).

[19] G. D’Amico, C. de Rham, S. Dubovsky, G. Gabadadze, D. Pirtskhalava, and A. J. Tolley, Massive cosmologies, Phys. Rev. D 84, 124046 (2011).

[20] M. von Strauss, A. Schmidt-May, J. Enander, E. Moertsell, and S.F. Hassan, Cosmological solutions in bimetric gravity and their observational tests, J. Cosmol. Astropart. Phys. 03 (2012) 042. 
[21] D. Comelli, M. Crisostomi, F. Nesti, and L. Pilo, FRW cosmology in ghost free massive gravity, J. High Energy Phys. 03 (2012) 067.

[22] Y. Akrami, T. S. Koivisto, and M. Sandstad, Accelerated expansion from ghost-free bigravity: A statistical analysis with improved generality, J. High Energy Phys. 03 (2013) 099.

[23] M. S. Volkov, Cosmological solutions with massive gravitons in the bigravity theory, J. High Energy Phys. 01 (2012) 035.

[24] F. Koennig, A. Patil, and L. Amendola, Viable cosmological solutions in massive bimetric gravity, J. Cosmol. Astropart. Phys. 03 (2014) 029.

[25] A. De Felice, A. E. Glu, S. Mukohyama, N. Tanahashi, and T. Tanaka, Viable cosmology in bimetric theory, J. Cosmol. Astropart. Phys. 06 (2014) 037.

[26] F. Könnig, Higuchi ghosts and gradient instabilities in bimetric gravity, Phys. Rev. D 91, 104019 (2015).

[27] M. Platscher, J. Smirnov, S. Meyer, and M. Bartelmann, Long range effects in gravity theories with Vainshtein screening, J. Cosmol. Astropart. Phys. 12 (2018) 009.

[28] E. Babichev, L. Marzola, M. Raidal, A. Schmidt-May, F. Urban, H. Veermäe, and M. von Strauss, Bigravitational origin of dark matter, Phys. Rev. D 94, 084055 (2016).

[29] E. Babichev, L. Marzola, M. Raidal, A. Schmidt-May, F. Urban, H. Veermae, and M. von Strauss, Heavy spin-2 dark matter, J. Cosmol. Astropart. Phys. 09 (2016) 016.

[30] X. Chu and C. Garcia-Cely, Self-interacting spin-2 dark matter, Phys. Rev. D 96, 103519 (2017).

[31] D. Comelli, M. Crisostomi, and L. Pilo, Perturbations in massive gravity cosmology, J. High Energy Phys. 06 (2012) 085.

[32] F. Koennig and L. Amendola, Instability in a minimal bimetric gravity model, Phys. Rev. D 90, 044030 (2014).

[33] F. Koennig, Y. Akrami, L. Amendola, M. Motta, and A. R. Solomon, Stable and unstable cosmological models in bimetric massive gravity, Phys. Rev. D 90, 124014 (2014).

[34] M. Lagos and P. G. Ferreira, Cosmological perturbations in massive bigravity, J. Cosmol. Astropart. Phys. 12 (2014) 026.

[35] Y. Akrami, S. F. Hassan, and F. Koennig, A. Schmidt-May, and A. R. Solomon, Bimetric gravity is cosmologically viable, Phys. Lett. B 748, 37 (2015).

[36] C. de Rham, A. J. Tolley, and D. H. Wesley, Vainshtein mechanism in binary pulsars, Phys. Rev. D 87, 044025 (2013).

[37] E. Babichev and C. Deffayet, An introduction to the Vainshtein mechanism, Classical Quantum Gravity 30, 184001 (2013).

[38] E. Babichev and M. Crisostomi, Restoring general relativity in massive bigravity theory, Phys. Rev. D 88, 084002 (2013).

[39] J. Enander and E. Mortsell, On stars, galaxies and black holes in massive bigravity, J. Cosmol. Astropart. Phys. 11 (2015) 023.

[40] C. de Rham and L. Heisenberg, Cosmology of the Galileon from massive gravity, Phys. Rev. D 84, 043503 (2011).

[41] N. Chow and J. Khoury, Galileon cosmology, Phys. Rev. D 80, 024037 (2009).
[42] A. G. Riess et al., A $2.4 \%$ determination of the local value of the Hubble constant, Astrophys. J. 826, 56 (2016).

[43] A. G. Riess et al., Milky Way Cepheid standards for measuring cosmic distances and application to Gaia DR2: Implications for the Hubble constant, Astrophys. J. 861, 126 (2018).

[44] H0LiCOW Collaboration, H0LiCOW V. New COSMOGRAIL time delays of HE 0435-1223: $H_{0}$ to $3.8 \%$ precision from strong lensing in a flat $\Lambda$ CDM model, Mon. Not. R. Astron. Soc. 465, 4914 (2017).

[45] H0LICOW Collaboration, H0LiCOW IX. Cosmographic analysis of the doubly imaged quasar SDSS $1206+$ 4332 and a new measurement of the Hubble constant, Mon. Not. R. Astron. Soc. 484, 4726 (2018).

[46] Planck Collaboration, Planck 2015 results. XIII. Cosmological parameters, Astron. Astrophys. 594, A13 (2016).

[47] Planck Collaboration, Planck 2018 results. VI. Cosmological parameters, Astron. Astrophys. 641, A6 (2020).

[48] G. Efstathiou, H0 revisited, Mon. Not. R. Astron. Soc. 440, 1138 (2014).

[49] G. E. Addison, Y. Huang, D. J. Watts, C. L. Bennett, M. Halpern, G. Hinshaw, and J. L. Weiland, Quantifying discordance in the 2015 Planck CMB spectrum, Astrophys. J. 818, 132 (2016).

[50] Planck Collaboration, Planck intermediate results. LI. Features in the cosmic microwave background temperature power spectrum and shifts in cosmological parameters, Astron. Astrophys. 607, A95 (2017).

[51] K. Aylor, M. K. Joy, L. Knox, M. Millea, S. Raghunathan, and W. L. K. Wu, Sounds discordant: Classical distance ladder \& $\Lambda$ CDM-based determinations of the cosmological sound horizon, Astrophys. J. 874, 4 (2019).

[52] E. Mörtsell and S. Dhawan, Does the Hubble constant tension call for new physics?, J. Cosmol. Astropart. Phys. 09 (2018) 025.

[53] Planck Collaboration, Planck intermediate results. XLVI. Reduction of large-scale systematic effects in HFI polarization maps and estimation of the reionization optical depth, Astron. Astrophys. 596, A107 (2016).

[54] B. Follin and L. Knox, Insensitivity of the distance ladder Hubble constant determination to Cepheid calibration modelling choices, Mon. Not. R. Astron. Soc. 477, 4534 (2018).

[55] S. Dhawan, S. W. Jha, and B. Leibundgut, Measuring the Hubble constant with type Ia supernovae as near-infrared standard candles, Astron. Astrophys. 609, A72 (2018).

[56] A. Schmidt-May and M. von Strauss, Recent developments in bimetric theory, J. Phys. A 49, 183001 (2016).

[57] K. Hinterbichler, Theoretical aspects of massive gravity, Rev. Mod. Phys. 84, 671 (2012).

[58] S. F. Hassan, A. Schmidt-May, and M. von Strauss, On consistent theories of massive spin-2 fields coupled to gravity, J. High Energy Phys. 05 (2013) 086.

[59] M. Lüben, A. Schmidt-May, and J. Weller, Physical parameter space of bimetric theory and SN1a constraints, J. Cosmol. Astropart. Phys. 09 (2020) 024.

[60] G. Cusin, R. Durrer, P. Guarato, and M. Motta, A general mass term for bigravity, J. Cosmol. Astropart. Phys. 04 (2016) 051. 
[61] M. Fasiello and A. J. Tolley, Cosmological stability bound in massive gravity and bigravity, J. Cosmol. Astropart. Phys. 12 (2013) 002.

[62] J. Enander and E. Moertsell, Strong lensing constraints on bimetric massive gravity, J. High Energy Phys. 10 (2013) 031.

[63] M. Platscher and J. Smirnov, Degravitation of the cosmological constant in bigravity, J. Cosmol. Astropart. Phys. 03 (2017) 051.

[64] M. Lüben, E. Mörtsell, and A. Schmidt-May, Bimetric cosmology is compatible with local tests of gravity, Classical Quantum Gravity 37, 047001 (2020).

[65] K. Aoki, K-. Maeda, and R. Namba, Stability of the early universe in bigravity theory, Phys. Rev. D 92, 044054 (2015).

[66] M. Högas, F. Torsello, and E. Moertsell, On the stability of bimetric structure formation, J. Cosmol. Astropart. Phys. 04 (2020) 046.

[67] E. Mortsell and J. Enander, Scalar instabilities in bimetric gravity: The Vainshtein mechanism and structure formation, J. Cosmol. Astropart. Phys. 10 (2015) 044.

[68] D. W. Pesce et al., The megamaser cosmology project. XIII. Combined Hubble constant constraints, Astrophys. J. Lett. 891, L1 (2020).

[69] S. M. Feeney, D. J. Mortlock, and N. Dalmasso, Clarifying the Hubble constant tension with a Bayesian hierarchical model of the local distance ladder, Mon. Not. R. Astron. Soc. 476, 3861 (2018).

[70] Planck Collaboration, Planck 2015 results. XIV. Dark energy and modified gravity, Astron. Astrophys. 594, A14 (2016).

[71] S. W. Hawking and G. F. R. Ellis, The large scale structure of space-time.

[72] R. R. Caldwell, A phantom menace?, Phys. Lett. B 545, 23 (2002).

[73] R. R. Caldwell, M. Kamionkowski, and N. N. Weinberg, Phantom Energy and Cosmic Doomsday, Phys. Rev. Lett. 91, 071301 (2003).

[74] P. H. Frampton and T. Takahashi, The fate of dark energy, Phys. Lett. B 557, 135 (2003).

[75] S. Nesseris and L. Perivolaropoulos, The fate of bound systems in phantom and quintessence cosmologies, Phys. Rev. D 70, 123529 (2004).

[76] S. M. Carroll, M. Hoffman, and M. Trodden, Can the dark energy equation-of-state parameter $w$ be less than -1 ?, Phys. Rev. D 68, 023509 (2003).

[77] S'. Nojiri, S. D. Odintsov, and S. Tsujikawa, Properties of singularities in (phantom) dark energy universe, Phys. Rev. D 71, 063004 (2005).

[78] S'. Nojiri and S. D. Odintsov, Inhomogeneous equation of state of the Universe: Phantom era, future singularity and crossing the phantom barrier, Phys. Rev. D 72, 023003 (2005).

[79] H. Stefancic, Expansion around the vacuum equation of state-Sudden future singularities and asymptotic behavior, Phys. Rev. D 71, 084024 (2005).

[80] N. Arkani-Hamed, H.-C. Cheng, M. A. Luty, and S. Mukohyama, Ghost condensation and a consistent infrared modification of gravity, J. High Energy Phys. 05 (2003) 074.

[81] V. Baccetti, P. Martin-Moruno, and M. Visser, Null energy condition violations in bimetric gravity, J. High Energy Phys. 08 (2012) 148.

[82] M. Lindner, K. Max, M. Platscher, and J. Rezacek, Probing alternative cosmologies through the inverse distance ladder, J. Cosmol. Astropart. Phys. 10 (2020) 040.

[83] M. C. March, R. Trotta, P. Berkes, G. D. Starkman, and P. M. Vaudrevange, Improved constraints on cosmological parameters from SNIa data, Mon. Not. R. Astron. Soc. 418, 2308 (2011).

[84] Supernova Search Team, Observational evidence from supernovae for an accelerating universe and a cosmological constant, Astron. J. 116, 1009 (1998).

[85] DES Collaboration, First cosmological results using type Ia supernovae from the dark energy survey: Measurement of the Hubble constant, Mon. Not. R. Astron. Soc. 486, 2184 (2019).

[86] Y. Kang, Y.-W. Lee, Y.-L. Kim, C. Chung, and C. H. Ree, Early-type host galaxies of type Ia supernovae. II. Evidence for luminosity evolution in supernova cosmology, arXiv:1912.04903.

[87] J. Colin, R. Mohayaee, M. Rameez, and S. Sarkar, Evidence for anisotropy of cosmic acceleration, Astron. Astrophys. 631, L13 (2019).

[88] D. J. Eisenstein and W. Hu, Baryonic features in the matter transfer function, Astrophys. J. 496, 605 (1998).

[89] F. Beutler, C. Blake, M. Colless, D. H. Jones, L. StaveleySmith, L. Campbell, Q. Parker, W. Saunders, and F. Watson, The 6dF Galaxy Survey: Baryon acoustic oscillations and the local Hubble constant, Mon. Not. R. Astron. Soc. 416, 3017 (2011).

[90] SDSS Collaboration, The clustering of galaxies in the SDSS-III baryon oscillation spectroscopic survey: Baryon acoustic oscillations in the data releases 10 and 11 galaxy samples, Mon. Not. R. Astron. Soc. 441, 24 (2014).

[91] N. Sasankan, M. R. Gangopadhyay, G. J. Mathews, and M. Kusakabe, Limits on brane-world and particle dark radiation from big bang nucleosynthesis and the CMB, Int. J. Mod. Phys. E 26, 1741007 (2017).

[92] N. Said, C. Baccigalupi, M. Martinelli, A. Melchiorri, and A. Silvestri, New constraints on the dark energy equation of state, Phys. Rev. D 88, 043515 (2013).

[93] DESI Collaboration, The DESI Experiment, a whitepaper for Snowmass 2013, arXiv:1308.0847.

[94] DESI Collaboration, The Dark Energy Spectroscopic Instrument (DESI), arXiv:1907.10688.

[95] D. J. Schlegel et al., Astro2020 APC White Paper: The MegaMapper: A $z>2$ spectroscopic instrument for the study of inflation and dark energy, arXiv:1907.11171.

[96] DESI Collaboration, The DESI experiment part I: Science, targeting, and survey design, arXiv:1611.00036.

[97] C. S. Kochanek, Rebuilding the Cepheid distance scale I: A global analysis of Cepheid mean magnitudes, Astrophys. J. 491, 13 (1997).

[98] L. M. Macri, K. Z. Stanek, D. Bersier, L. Greenhill, and M. Reid, A new Cepheid distance to the maser-host galaxy 
NGC 4258 and its implications for the Hubble constant, Astrophys. J. 652, 1133 (2006).

[99] J. R. Gerke, C. S. Kochanek, J. L. Prieto, K. Z. Stanek, and L. M. Macri, A study of Cepheids in M81 with the large binocular telescope (efficiently calibrated with HST), Astrophys. J. 743, 176 (2011).

[100] A. Higuchi, Forbidden mass range for spin-2 field theory in de Sitter space-time, Nucl. Phys. B282, 397 (1987). 